\title{
Complexity-Reduced MLD Based on QR Decomposition in OFDM MIMO Multiplexing with Frequency Domain Spreading and Code Multiplexing
}

\author{
Kouji Nagatomi, ${ }^{1}$ Hiroyuki Kawai, ${ }^{2}$ and Kenichi Higuchi ${ }^{1}$ \\ ${ }^{1}$ Department of Electrical Engineering, Tokyo University of Science, 2641 Yamazaki, Noda, Chiba 278-8510, Japan \\ ${ }^{2}$ Radio Access Network Development Department, NTT DOCOMO, INC., 3-5 Hikari-no-oka, Yokosuka, Kanagawa 239-8536, Japan
}

Correspondence should be addressed to Kenichi Higuchi, higuchik@rs.noda.tus.ac.jp

Received 12 April 2010; Revised 30 June 2010; Accepted 19 August 2010

Academic Editor: Naofal Al-Dhahir

Copyright (C) 2011 Kouji Nagatomi et al. This is an open access article distributed under the Creative Commons Attribution License, which permits unrestricted use, distribution, and reproduction in any medium, provided the original work is properly cited.

\begin{abstract}
This paper presents a new maximum likelihood detection- (MLD-) based signal detection method for orthogonal frequency division multiplexing (OFDM) multiple-input multiple-output (MIMO) multiplexing with frequency domain spreading and code multiplexing. The proposed MLD reduces the computational complexity by utilizing signal orthogonalization based on QR decomposition of the product of the channel and spreading code matrices in the frequency domain. Simulation results show that when the spreading factor and number of code multiplexed symbols are 16, the proposed MLD reduces the average received signal energy per bit-to-noise spectrum density ratio $\left(E_{b} / N_{0}\right)$ for the average packet error rate (PER) of $10^{-2}$ by approximately $12 \mathrm{~dB}$ compared to the conventional minimum mean-squared error- (MMSE-) based filtering for 4-by-4 MIMO multiplexing (16QAM with the rate-3/4 Turbo code is assumed).
\end{abstract}

\section{Introduction}

Orthogonal frequency division multiplexing (OFDM) is a promising modulation/radio access scheme for future wireless communication systems because of its inherent immunity to multipath interference due to a low symbol rate and the use of a cyclic prefix (CP), and its affinity to different transmission bandwidth arrangements. OFDM has already been adopted as a radio access scheme for several of the latest cellular system specifications such as the longterm evolution (LTE) system in the 3GPP (3rd Generation Partnership Project) [1].

One of the major drawbacks of the OFDM signal based on multicarrier transmission is the high peak-to-average power ratio (PAPR) of the transmit signal. The OFDM signal also cannot achieve symbol-level multipath diversity (frequency diversity in the frequency domain) since each of the narrow-band subcarriers experiences flat fading variation even in multipath fading environments, although some frequency diversity gain is obtained by using channel coding.
One approach to achieve a lower PAPR and multipath diversity gain in the OFDM signal is to use frequency domain spreading and code multiplexing (in other words, linear precoding before the inverse fast Fourier transform (IFFT) modulation at the transmitter) [2-9]. Code multiplexing is needed if we want to maintain the same frequency efficiency as that without frequency domain spreading. In general, by using the frequency domain spreading at the transmitter and frequency domain despreading at the receiver, symbol-level frequency domain diversity is achieved in a multipath fading channel [2-5]. Furthermore, by selecting an appropriate set of spreading codes, frequency domain spreading and code multiplexing in the OFDM signal can reduce the PAPR [6-9]. In particular, when the discrete Fourier transform (DFT) sequence is used as a spreading code, which is called DFT-Spread OFDM [1, 8, 9], a very low PAPR, which is the same as that of the single carrier transmission, is achieved.

In general, the use of frequency domain spreading and code multiplexing, however, loses the inherent immunity 
of the OFDM signal to multipath interference. Thus, intersymbol interference (ISI) occurs in a multipath fading channel. The ISI between code-multiplexed symbols degrades the transmission quality of the OFDM signal with frequency domain spreading and code multiplexing especially when space division multiplexing (SDM; hereafter referred to as multiple-input multiple-output (MIMO) multiplexing) [10] is applied to achieve a high data rate.

The use of frequency domain spreading and code multiplexing also restricts the use of powerful signal detection methods. Maximum likelihood detection (MLD) is known as an optimum signal detection scheme for MIMO multiplexing [11]. However, when frequency domain spreading and code multiplexing is applied to the OFDM signal, the number of symbol candidates is exponentially increased to $2^{N_{\mathrm{R}} N_{\mathrm{TX}} N_{\mathrm{SF}}}$, where $N_{\mathrm{R}}$ is the number of bits conveyed by one symbol, $N_{\mathrm{TX}}$ is the number of the transmitter antennas, and $N_{\mathrm{SF}}$ is the spreading factor that equals the number of code multiplexed symbols. Therefore, the use of MLD is not realistic and a low-complexity signal detector such as linear filtering based on the minimum mean-squared error (MMSE) must be used. This is another reason why the bit error rate (BER) and packet error rate (PER) of MIMO multiplexing with the OFDM signal using frequency domain spreading and code multiplexing are deteriorated compared to that of OFDM MIMO multiplexing without spreading.

This paper presents a new MLD-based signal detection method for OFDM MIMO multiplexing with frequency domain spreading and code multiplexing. The proposed MLD-based signal detection method is based on the QR decomposition- (QRD-) M algorithm [12] (or QRM-MLD in [13]) for OFDM MIMO multiplexing, which applies signal orthogonalization based on $\mathrm{QR}$ decomposition of the spatial channel matrix and quasi-MLD using the computationally efficient $M$-algorithm on the orthogonalized signal for each subcarrier independently. However, when we assume frequency domain spreading and code multiplexing, the signal constellation per transmitter antenna still has $2^{N_{\mathrm{R}} N_{\mathrm{SF}}}$ points although the spatially multiplexed symbols are decomposed if we employ the per subcarrier-based QRD- $M$ or QRM-MLD. Therefore, in order to decompose fully the spatial and code multiplexed transmit symbols at the receiver, the proposed MLD receiver jointly considers all the subcarriers to which the spread symbols are mapped and constructs the overall frequency-domain linear transformation matrix, which is a product of the space and frequencydomain channel matrix and spreading code matrix. The QR decomposition of the overall frequency-domain linear transformation matrix is performed to derive the orthogonalized received signal vector. Then, the $M$-algorithm is used to achieve computationally efficient quasi-MLD with the orthogonalized received signal vector. We note that the MMSE-based Turbo equalization, for example, in [14-17], is another powerful candidate for signal detection for OFDM MIMO multiplexing with frequency domain spreading and code multiplexing. A possible advantageous property of the proposed MLD against the MMSE-based Turbo equalization can be a shorter processing delay as the proposed MLD does not require iterative signal detection and Turbo decoding which is different from Turbo equalization. The computational complexity of the proposed MLD may be higher than that of the MMSE-based Turbo equalization as $N_{\mathrm{SF}}$ increases. A detailed comparison of the proposed MLD and the MMSEbased Turbo equalization is outside the scope of the paper and is left for future study.

In the paper, we also propose a spreading code-first ordering method of spatial/code-multiplexed symbols that are to be detected in order to decrease the symbol selection error in the proposed MLD due to the fading correlation between the code-multiplexed symbols transmitted from the same transmitter antenna. The reminder of the paper is organized as follows. First, Section 2 describes the proposed MLD-based signal detection method. Then in Section 3, we present a set of simulation results to show the PER improvement when using the proposed MLD compared to the MMSE-based linear filtering. Finally, Section 4 concludes the paper.

\section{Complexity-Reduced MLD for OFDM MIMO Multiplexing with Frequency Domain Spreading and Code Multiplexing}

2.1. Basic Structure of Proposed MLD. Figure 1 shows a block diagram of the OFDM MIMO transmitter using frequency domain spreading and code multiplexing. In the following, we assume that the number of subcarriers of interest is equal to the spreading factor, $N_{\mathrm{SF}}$, for the sake of simplicity. Furthermore, we assume that the number of code multiplexing is equal to $N_{\mathrm{SF}}$ in order to maintain the same frequency efficiency as that without frequency domain spreading.

The $N_{\mathrm{SF}} \times 1$-dimensional transmit data symbol vector, $\mathbf{s}_{n}$, which will be spread and code-multiplexed later, from the $n$th $\left(1 \leq n \leq N_{\mathrm{TX}}\right)$ transmit antenna is represented as

$$
\mathbf{s}_{n}=\left[\begin{array}{llll}
s_{n, 1} & s_{n, 2} & \cdots & s_{n, N_{\mathrm{SF}}}
\end{array}\right]^{t},
$$

where $s_{n, b}$ is the $b$ th $\left(1 \leq b \leq N_{\mathrm{SF}}\right)$ data symbol from the $n$th transmit antenna and $(\cdot)^{t}$ is the transpose operation. The $N_{\mathrm{SF}} \times 1$-dimensional spreading code sequence vector, $\mathbf{w}_{i}$, each of whose elements is multiplied to each data symbol at the $i$ th $\left(1 \leq i \leq N_{\mathrm{SF}}\right)$ subcarrier, is expressed as

$$
\mathbf{w}_{i}=\left[\begin{array}{llll}
w_{i, 1} & w_{i, 2} & \cdots & w_{i, N_{\mathrm{SF}}}
\end{array}\right]^{t},
$$

where $w_{i, b}$ is the spreading code multiplied to the $b$ th data symbol at the $i$ th subcarrier. Spreading code sequence vector $\mathbf{w}_{i}$ is the $i$ th column vector of the $N_{\mathrm{SF}} \times N_{\mathrm{SF}}$-dimensional spreading code matrix, $\mathbf{W}$. In general, a unitary matrix is used as $\mathbf{W}$. Since we assume DFT-Spread OFDM in the following evaluation, each of the column vectors of the 


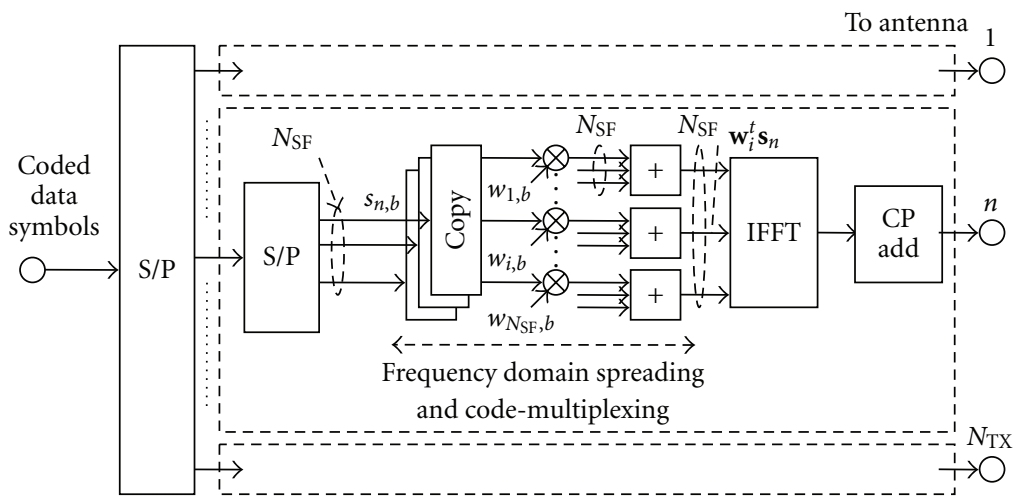

FIGURE 1: Block diagram of the OFDM MIMO transmitter using frequency domain spreading and code multiplexing.

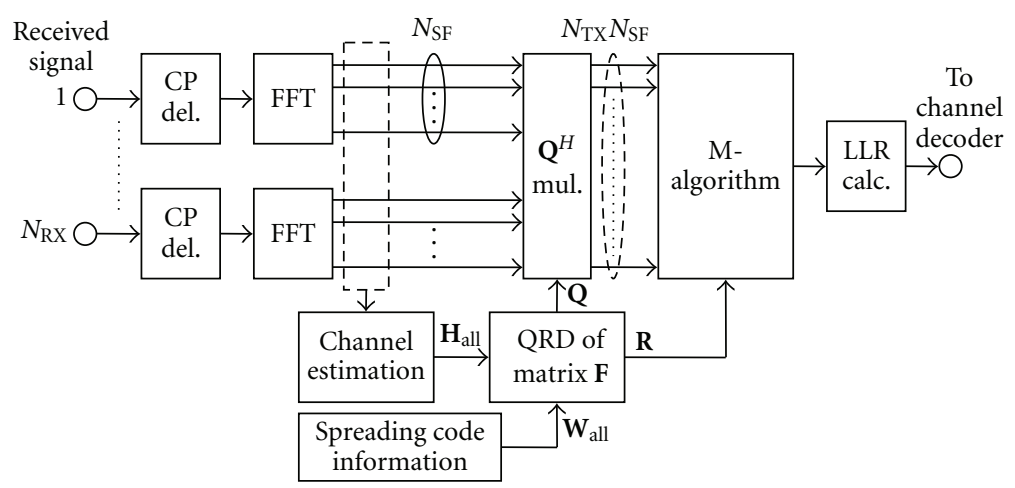

FIGURE 2: Block diagram of the proposed MLD-based signal detection.

$N_{\mathrm{SF}} \times N_{\mathrm{SF}}$-dimensional DFT matrix, $\mathbf{W}_{\mathrm{DFT}}$, is used as $\mathbf{w}_{i}$ in the paper:

$$
\begin{aligned}
& \mathbf{W}_{\mathrm{DFT}}=\left[\begin{array}{llll}
\mathbf{w}_{1} & \mathbf{w}_{2} & \cdots & \mathbf{w}_{N_{\mathrm{SF}}}
\end{array}\right] \\
& =\left[\begin{array}{c}
\tilde{\mathbf{w}}_{1} \\
\tilde{\mathbf{w}}_{2} \\
\vdots \\
\tilde{\mathbf{w}}_{N_{\mathrm{SF}}}
\end{array}\right] \\
& =\frac{1}{\sqrt{N_{\mathrm{SF}}}}\left[\phi_{N_{\mathrm{SF}}}(l-1)(i-1)\right],
\end{aligned}
$$

where $\phi_{N_{\mathrm{SF}}}=e^{-j 2 \pi / N_{\mathrm{SF}}}$, and $l$ and $i$ represent the index for the rows and columns of $\mathbf{W}_{\mathrm{DFT}}$, respectively $\left(1 \leq l, i \leq N_{\mathrm{SF}}\right)$. The $1 \times N_{\mathrm{SF}}$-dimensional spreading code sequence vector, $\tilde{\mathbf{w}}_{b}$, can be seen as a spreading code sequence for the $b$ th data symbol. It should be noted that the same matrix, $\mathbf{W}_{\mathrm{DFT}}$, is commonly used for spreading at all the transmitter antennas. The transmit signal from the $n$th transmit antenna at the $i$ th subcarrier is represented as $\mathbf{w}_{i}^{t} \mathbf{s}_{n}$. The frequency-domain transmit signal is converted to a time-domain transmit signal by inverse fast Fourier transform (IFFT) operation and transmitted after appending a CP.

We define $N_{\mathrm{RX}} \times N_{\mathrm{TX}}$-dimensional matrix $\mathbf{H}_{i}$ assuming that $N_{\mathrm{RX}}$ is the number of receiver antennas, which comprises channel coefficients for all the combinations of transmitter and receiver antennas for the $i$ th subcarrier:

$$
\mathbf{H}_{i}=\left[\begin{array}{cccc}
h_{i, 1,1} & h_{i, 1,2} & \cdots & h_{i, 1, N_{\mathrm{TX}}} \\
h_{i, 2,1} & h_{i, 2,2} & & \\
\vdots & & \ddots & \\
h_{i, N_{\mathrm{RX}}, 1} & & & h_{i, N_{\mathrm{RX}}, N_{\mathrm{TX}}}
\end{array}\right] .
$$

Here $h_{i, m, n}$ denotes the channel coefficient between the $n$th transmit antenna and the $m$ th $\left(1 \leq m \leq N_{\mathrm{RX}}\right)$ receiver antenna at the $i$ th subcarrier.

At the receiver, after the CP removal, the time-domain received signal is converted to a frequency-domain signal by FFT operation at each receiver antenna branch. Assuming that the time difference in the propagation delay of all the multipaths is within the CP duration, the $N_{\mathrm{RX}} \times 1$ dimensional frequency-domain received signal vector, $\mathbf{r}_{i}$, for the $i$ th subcarrier is represented as

$$
\begin{aligned}
\mathbf{r}_{i} & =\mathbf{H}_{i}\left[\begin{array}{c}
\mathbf{w}_{i}^{t} \mathbf{s}_{1} \\
\mathbf{w}_{i}^{t} \mathbf{s}_{2} \\
\vdots \\
\mathbf{w}_{i}^{t} \mathbf{s}_{N_{\mathrm{TX}}}
\end{array}\right]+\mathbf{n}_{i} \\
& =\mathbf{H}_{i} \operatorname{diag}\left\{\mathbf{w}_{i}^{t}\right\}\left[\begin{array}{llll}
\mathbf{s}_{1}^{t} & \mathbf{s}_{2}^{t} & \cdots & \mathbf{s}_{N_{\mathrm{TX}}}^{t}
\end{array}\right]^{t}+\mathbf{n}_{i} \\
& =\mathbf{H}_{i} \mathbf{W}_{i} \mathbf{s}_{\text {all }}+\mathbf{n}_{i},
\end{aligned}
$$




$$
\begin{gathered}
\mathbf{W}_{i}=\operatorname{diag}\left\{\mathbf{w}_{i}^{t}\right\}, \\
\mathbf{s}_{\text {all }}=\left[\begin{array}{llll}
\mathbf{s}_{1}^{t} & \mathbf{s}_{2}^{t} & \cdots & \mathbf{s}_{\mathrm{NT}_{\mathrm{TX}}}^{t}
\end{array}\right]^{t},
\end{gathered}
$$

where $\operatorname{diag}\left\{\mathbf{w}_{i}^{t}\right\}$ is the $N_{\mathrm{TX}} \times N_{\mathrm{TX}} N_{\mathrm{SF}}$-dimensional block diagonal matrix all of whose block diagonal components are $\mathbf{w}_{i}^{t}$ and hereafter is simply denoted as $\mathbf{W}_{i}$. The $N_{\mathrm{TX}} N_{\mathrm{SF}} \times 1-$ dimensional vector, $\mathbf{s}_{\text {all }}$, is the overall transmit data symbol vector whose $\left((n-1) N_{\mathrm{SF}}+b\right)$ th element represents the $b$ th data symbol transmitted from the $n$th transmit antenna. Vector $\mathbf{n}_{i}$ is an $N_{\mathrm{RX}} \times 1$-dimensional receiver noise vector assuming i.i.d. additive white Gaussian noise (AWGN).

The overall frequency-domain received signal vector is represented as

$$
\begin{aligned}
\mathbf{r}_{\text {all }} & =\left[\begin{array}{c}
\mathbf{r}_{1} \\
\mathbf{r}_{2} \\
\vdots \\
\mathbf{r}_{\mathrm{SF}_{\mathrm{SF}}}
\end{array}\right]=\left[\begin{array}{c}
\mathbf{H}_{1} \mathbf{W}_{1} \\
\mathbf{H}_{2} \mathbf{W}_{2} \\
\vdots \\
\mathbf{H}_{N_{\mathrm{SF}}} \mathbf{W}_{N_{\mathrm{SF}}}
\end{array}\right] \mathbf{s}_{\text {all }}+\left[\begin{array}{c}
\mathbf{n}_{1} \\
\mathbf{n}_{2} \\
\vdots \\
\mathbf{n}_{N_{\mathrm{SF}}}
\end{array}\right] \\
& =\mathbf{H}_{\text {all }} \mathbf{W}_{\text {all }} \mathbf{S}_{\text {all }}+\mathbf{n}_{\mathrm{all}} \\
& =\mathbf{F s}_{\text {all }}+\mathbf{n}_{\mathrm{all}}, \\
\mathbf{H}_{\text {all }} & =\operatorname{diag}\left[\begin{array}{llll}
\mathbf{H}_{1} & \mathbf{H}_{2} & \cdots & \mathbf{H}_{N_{\mathrm{SF}}}
\end{array}\right], \\
\mathbf{W}_{\text {all }} & =\left[\begin{array}{llll}
\mathbf{W}_{1}^{t} & \mathbf{W}_{2}^{t} & \cdots & \mathbf{W}_{N_{\mathrm{SF}}}^{t}
\end{array}\right]^{t}, \\
\mathbf{n}_{\text {all }} & =\left[\begin{array}{llll}
\mathbf{n}_{1}^{t} & \mathbf{n}_{2}^{t} & \cdots & \mathbf{n}_{N_{\mathrm{SF}}}^{t}
\end{array}\right]^{t}, \\
\mathbf{F} & =\mathbf{H}_{\text {all }} \mathbf{W}_{\mathrm{all}},
\end{aligned}
$$

where F denotes the matrix of size $N_{\mathrm{RX}} N_{\mathrm{SF}} \times N_{\mathrm{TX}} N_{\mathrm{SF}}$, which comprises the product of the extended channel matrix and spreading code matrix in the frequency domain.

In the proposed MLD-based signal detection, $\mathbf{F}$ is estimated at the receiver from the channel estimate and known spreading code matrix. Next, QR decomposition is performed on the estimated $\widetilde{\mathbf{F}}$ :

$$
\widetilde{\mathbf{F}} \Longrightarrow \mathbf{Q R} \text {. }
$$

Matrix $\mathbf{Q}$ is an $N_{\mathrm{RX}} N_{\mathrm{SF}} \times N_{\mathrm{TX}} N_{\mathrm{SF}}$-dimensional unitary matrix and $\mathbf{R}$ is an $N_{\mathrm{TX}} N_{\mathrm{SF}} \times N_{\mathrm{TX}} N_{\mathrm{SF}}$-dimensional upper triangular matrix. Assuming that $\widetilde{\mathbf{F}}$ has no estimation error, the orthogonalization of the received signal vector is achieved by multiplying the Hermitian transpose of matrix $\mathbf{Q}$ to the overall frequency-domain received signal vector:

$$
\begin{aligned}
\mathbf{z} & =\mathbf{Q}^{H} \mathbf{r}_{\text {all }}=\mathbf{Q}^{H}\left(\mathbf{F s}_{\text {all }}+\mathbf{n}_{\text {all }}\right) \\
& =\mathbf{Q}^{H}\left(\mathbf{Q R} \mathbf{s}_{\text {all }}+\mathbf{n}_{\text {all }}\right)=\mathbf{R} \mathbf{s}_{\text {all }}+\mathbf{Q}^{H} \mathbf{n}_{\text {all }} .
\end{aligned}
$$

Here $(\cdot)^{H}$ denotes the Hermitian transpose operation. Vector $\mathbf{z}$ is the $N_{\mathrm{TX}} N_{\mathrm{SF}} \times 1$-dimensional orthogonalized received signal vector. Since matrix $\mathbf{Q}$ is unitary, the transformed $N_{\mathrm{TX}} N_{\mathrm{SF}} \times 1$-dimensional receiver noise vector $\mathbf{Q}^{H} \mathbf{n}_{\text {all }}$ still maintains the i.i.d. AWGN property.
Several kinds of complexity-reduced MLD-based signal detection methods can be applied to orthogonalized received signal vector $\mathbf{z}$ such as the $M$-algorithm $[12,13]$, sphere decoding [18], or stack algorithm [19]. In the paper, we use the $M$-algorithm. It should be noted that we can use the MMSE-based QR decomposition [20] by extending matrix F considering the receiver noise power. By applying the MMSE-based QR decomposition, it can be expected that the number of false discards of the correct symbol candidates especially at the earlier stages of the $M$-algorithm will be decreased. However, we use zero forcing- (ZF-) based QR decomposition as in (13) in the following evaluation for the sake of simplicity.

Figure 2 shows the receiver block diagram of the proposed MLD. The number of stages in the $M$-algorithm is $N_{\mathrm{TX}} N_{\mathrm{SF}}$. The $M$-algorithm keeps only $M$ candidate symbol vectors that have the highest reliability at each stage. Let $\widehat{\boldsymbol{s}}_{q}^{(k)}(1 \leq q \leq M)$ be the $q$ th $k \times 1$-dimensional surviving candidate symbol vector at the $k$ th stage, which contains the $N_{\mathrm{TX}} N_{\mathrm{SF}}-k+1$ to the $\left(N_{\mathrm{TX}} N_{\mathrm{SF}}\right)$ th elements of $\mathbf{s}_{\text {all }}$. Then, the $(k+1)$ th stage has $M 2^{N_{\mathrm{R}}}$ candidate symbol vectors to be evaluated. Each of them is represented as

$$
\widetilde{\mathbf{s}}_{p, q}^{(k+1)}=\left[\begin{array}{c}
c_{p} \\
\widehat{\mathbf{s}}_{q}^{(k)}
\end{array}\right],
$$

where $1 \leq p \leq 2^{N_{\mathrm{R}}}$ and $c_{p}$ represents the $p$ th complex symbol candidate. We define $(k+1) \times 1$-dimensional vector $\mathbf{z}^{(k+1)}$ and $(k+1) \times N_{\mathrm{TX}} N_{\mathrm{SF}}$-dimensional matrix $\mathbf{R}^{(k+1)}$ as follows:

$$
\begin{aligned}
\mathbf{z}^{(k+1)} & =\left[\begin{array}{llll}
z_{N_{\mathrm{TX}} N_{\mathrm{SF}}-k} & z_{\mathrm{N}_{\mathrm{TX}} N_{\mathrm{SF}}-k+1} & \cdots & z_{N_{\mathrm{TX}} N_{\mathrm{SF}}}
\end{array}\right]^{t}, \\
\mathbf{R}^{(k+1)} & =\left[\begin{array}{llll}
\mathbf{R}_{N_{\mathrm{TX}} N_{\mathrm{SF}}-k}^{t} & \mathbf{R}_{N_{\mathrm{TX}} N_{\mathrm{SF}}-k+1}^{t} & \cdots & \mathbf{R}_{N_{\mathrm{TX}} N_{\mathrm{SF}}}^{t}
\end{array}\right]^{t} .
\end{aligned}
$$

Here, $z_{j}$ and $\mathbf{R}_{j}$ are the $j$ th element of $\mathbf{z}$ and the $j$ th row vector of $\mathbf{R}$, respectively. The accumulated branch metric $\Lambda_{p, q}$ for the candidate symbol vector $\widetilde{\mathbf{s}}_{p, q}^{(k+1)}$ is calculated as

$$
\begin{aligned}
\Lambda_{p, q}= & \left\|\mathbf{z}^{(k+1)}-\mathbf{R}^{(k+1)}\left[\begin{array}{c}
\mathbf{0}_{N_{\mathrm{TX}} N_{\mathrm{SF}}-k-1} \\
\tilde{\mathbf{s}}_{p, q}^{(k+1)}
\end{array}\right]\right\|^{2} \\
= & \left\|z_{1}^{(k+1)}-\mathbf{R}_{1}^{(k+1)}\left[\begin{array}{c}
\mathbf{0}_{N_{\mathrm{TX}} N_{\mathrm{SF}}-k-1} \\
\tilde{\mathbf{s}}_{p, q}^{(k+1)}
\end{array}\right]\right\|^{2} \\
& +\left\|\mathbf{z}^{(k)}-\mathbf{R}^{(k)}\left[\begin{array}{c}
\mathbf{0}_{N_{\mathrm{TX}} N_{\mathrm{SF}}-k} \\
\hat{\mathbf{s}}_{q}^{(k)}
\end{array}\right]\right\|^{2},
\end{aligned}
$$

where $z_{1}^{(k+1)}$ and $\mathbf{R}_{1}^{(k+1)}$ are the first element of $\mathbf{z}^{(k+1)}$ and the first row vector of $\mathbf{R}^{(k+1)}$, respectively, and $\mathbf{0}_{x}$ is an $x \times 1$ dimensional vector all of whose elements are zero. It should be noted that the second term of (17) is calculated at the $k$ th stage and therefore it does not need to be calculated at the $(k+1)$ th stage. The $\widetilde{\mathbf{s}}_{p, q}^{(k+1)}$ are arranged from the one with the smallest accumulated branch metric in increasing order and $M$-best $\widetilde{\mathbf{s}}_{p, q}^{(k+1)}$ are selected as surviving candidate symbol vectors $\hat{\mathbf{s}}_{q}^{(k+1)}(1 \leq q \leq M)$ to the next stage. This process 


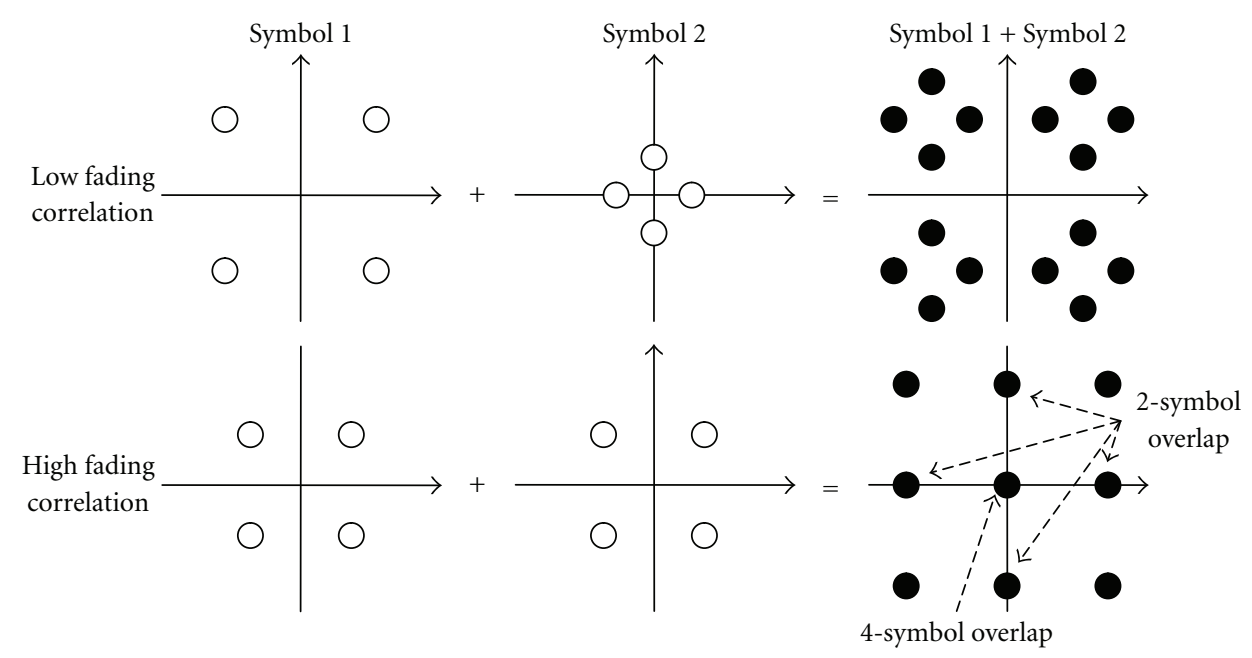

FIGURE 3: Impact of fading correlation on surviving symbol selection in $M$-algorithm (QPSK modulation is assumed).

is repeated for $N_{\mathrm{TX}} N_{\mathrm{SF}}$ stages. Therefore, the total number of branch metric calculations is reduced from $2^{N_{\mathrm{R}} N_{\mathrm{TX}} N_{\mathrm{SF}}}$, which is required for full MLD, to $M 2^{N_{\mathrm{R}}} N_{\mathrm{TX}} N_{\mathrm{SF}}$ by using the proposed MLD. Finally, the log likelihood ratio (LLR) for each channel coded bit is calculated from the branch metrics of the surviving symbol candidates at the last stage of the $M$-algorithm, and channel decoding is performed to recover the transmit data sequences.

2.2. Symbol Ordering in Proposed MLD. In the description of the proposed MLD in the previous subsection, we assumed that the transmit symbols are ordered in $\mathbf{s}_{\text {all }}$ so that the set of the code-multiplexed symbols from the same transmit antenna is located in the same neighborhood in (8). Thus, the $\left((n-1) N_{\mathrm{SF}}+b\right)$ th element of $\mathbf{s}_{\text {all }}$ is the $b$ th data symbol transmitted from the $n$th transmit antenna. However, this order can be arbitrarily changed at the receiver by exchanging the corresponding columns in matrix $\widetilde{\mathbf{F}}$. As is described in $[12,13]$, the ordering (ranking) of the symbols in which stage each symbol appears first affects the achievable PER of quasi-MLD based on the $M$-algorithm greatly since the $M$-algorithm successively reduces the number of symbol candidates stage-by-stage from the symbols mapped to the bottom of the transmit symbol vector. Therefore, we investigate the following two symbol ordering strategies for the proposed MLD.

2.2.1. Antenna-First Ordering Method. The received signal power used for the selection of the surviving symbol candidates for the $l$ th ordered symbols (thus, $\left(N_{\mathrm{TX}} N_{\mathrm{SF}}-\right.$ $l+1)$ th element of $\left.\mathbf{s}_{\text {all }}\right)$ at the $k(k \geq l)$-th stage of the $M$-algorithm is the sum of the square of the elements from $N_{\mathrm{TX}} N_{\mathrm{SF}}-k+1$ to the $\left(N_{\mathrm{TX}} N_{\mathrm{SF}}\right)$ th row at the $\left(N_{\mathrm{TX}} N_{\mathrm{SF}}-\right.$ $l+1)$ th column of $\mathbf{R}$. Therefore, the probability of false discard of the correct symbol candidates is greater at an earlier stage. The symbol ordering based on the received signal power or signal-to-interference and noise power ratio (SINR) of each symbol are presented in $[12,13]$ for the
OFDM case without spreading and code multiplexing. A symbol in good condition is set to be tested from an earlier stage. We call this method antenna-first ordering in the paper. It should be noted that since the received signal power of all code-multiplexed symbols from the same transmit antenna are the same assuming that each element of the spreading code matrix has the same power (this is true, e.g., in DFT and Walsh-Hadamard matrices), the antenna-first ordering method orders the symbols so that the set of the codemultiplexed symbols from each transmit antenna is blockwised as in (8). Assuming that the transmitter antenna branch indexes are arranged from the one with the smallest received signal power in increasing order, let $f(n)$ be the transmitter antenna branch index ranked at the $n$th order. Then, the $\left((f(n)-1) N_{\mathrm{SF}}+b\right)$ th column vector of the original form of $\mathbf{F}$ in $(12)$ is moved to the $\left((n-1) N_{\mathrm{SF}}+b\right)$ th column in the antenna-first ordering, so that the $b$ th data symbol transmitted from the $f(n)$ th transmit antenna becomes the $\left((n-1) N_{\mathrm{SF}}+b\right)$ th element of $\boldsymbol{s}_{\text {all }}$. The resultant $\mathbf{F}$ and $\boldsymbol{s}_{\text {all }}$ are represented, respectively, as

$$
\begin{aligned}
& \mathbf{F}=\left[\begin{array}{ccc}
h_{1,1, f(1)} \mathbf{w}_{1}^{t} & \cdots & h_{1,1, f\left(N_{\mathrm{TX}}\right)} \mathbf{w}_{1}^{t} \\
\vdots & \ddots & \vdots \\
h_{1, N_{\mathrm{RX}}, f(1) \mathbf{w}_{1}^{t}} & \cdots & h_{1, N_{\mathrm{RX}}, f\left(N_{\mathrm{TX}}\right)} \mathbf{w}_{1}^{t} \\
& \vdots & \\
h_{\mathrm{SF}_{\mathrm{SF}}, 1, f(1)} \mathbf{w}_{N_{\mathrm{SF}}}^{t} & \cdots & h_{N_{\mathrm{SF}}, 1, f\left(N_{\mathrm{TX}}\right)} \mathbf{w}_{N_{\mathrm{SF}}}^{t} \\
\vdots & \ddots & \vdots \\
h_{N_{\mathrm{SF}}, N_{\mathrm{RX}}, f(1)} \mathbf{w}_{N_{\mathrm{SF}}}^{t} & \cdots & h_{N_{\mathrm{SF}}, N_{\mathrm{RX}}, f\left(N_{\mathrm{TX}}\right)} \mathbf{w}_{N_{\mathrm{SF}}}^{t}
\end{array}\right], \\
& \mathbf{s}_{\mathrm{all}}=\left[\begin{array}{llll}
\mathbf{s}_{f(1)}^{t} & \mathbf{s}_{f(2)}^{t} & \cdots & \mathbf{s}_{f\left(N_{\mathrm{TX}}\right)}^{t}
\end{array}\right]^{t} .
\end{aligned}
$$

2.2.2. Code-First Ordering Method. The accuracy of the surviving symbol candidates is in general degraded in the $M$-algorithm for the combination of transmitted symbols with a high fading correlation. This is because multiple symbol candidates may have very similar branch metrics 
(similar squared Euclidian distances to the received signal point) in this case as shown in Figure 3.

In OFDM MIMO multiplexing with the frequency domain spreading and code multiplexing, the fading correlation among code-multiplexed symbols transmitted from the same transmit antenna is one. To see clearly the shape of the matrix $\mathbf{R}$ with the antenna-first ordering, let us assume flat fading here such as $\mathbf{H}_{1}=\mathbf{H}_{2}=\ldots=\mathbf{H}_{N_{\mathrm{SF}}}$. In this case, $(n-$ 1) $N_{\mathrm{SF}}+1$ to $n N_{\mathrm{SF}}$ th column vectors of matrix $\mathbf{F}$ in the form of (18) are orthogonal to each other since $\mathbf{W}$ is a unitary matrix, and every $N_{\mathrm{SF}}$ th column vector has correlation. Therefore, matrix $\mathbf{R}$ with the antenna-first ordering is represented as

$$
\mathbf{R}=\left[\begin{array}{ccccc}
\operatorname{diag}\left\{\lambda_{1,1}\right\} & \operatorname{diag}\left\{\lambda_{1,2}\right\} & \operatorname{diag}\left\{\lambda_{1,3}\right\} & \ldots & \operatorname{diag}\left\{\lambda_{1, N_{\mathrm{TX}}}\right\} \\
0 & \operatorname{diag}\left\{\lambda_{2,2}\right\} & \operatorname{diag}\left\{\lambda_{2,3}\right\} & \cdots & \operatorname{diag}\left\{\lambda_{2, N_{T X}}\right\} \\
& & \ddots & & \vdots \\
0 & & 0 & \operatorname{diag}\left\{\lambda_{N_{\mathrm{TX}}-1, N_{\mathrm{TX}}-1}\right\} & \operatorname{diag}\left\{\lambda_{N_{\mathrm{TX}}-1, N_{\mathrm{TX}}}\right\} \\
& & 0 & \operatorname{diag}\left\{\lambda_{N_{\mathrm{TX}}, N_{\mathrm{TX}}}\right\}
\end{array}\right]
$$

$\mathbf{W}$ is a unitary matrix. Therefore, matrix $\mathbf{R}$ with the codefirst ordering is represented as

$$
\mathbf{R}=\left[\begin{array}{ccccc}
\mathbf{R}_{\text {sub }} & 0 & & & 0 \\
0 & \mathbf{R}_{\text {sub }} & 0 & & \\
& 0 & \mathbf{R}_{\text {sub }} & & \\
& & 0 & \ddots & 0 \\
0 & & & 0 & \mathbf{R}_{\text {sub }}
\end{array}\right]
$$

where $\mathbf{R}_{\text {sub }}$ is the $N_{\mathrm{TX}} \times N_{\mathrm{TX}}$-dimensional upper triangular matrix. Thus, after orthogonalization, the signal components of the transmit symbol of interest appear in consecutive $N_{\text {TX }}$ stages using the code-first ordering. This makes surviving symbol replica selection accurate compared to the case with the antenna-first ordering. Similar to the case with antennafirst ordering, when the channel is frequency selective, all of the upper triangular elements of matrix $\mathbf{R}$, which are zero in (23), can take nonzero values.

We note that the code-first ordering method can additionally use the received signal power-based ordering with secondary priority. In this case, the elements of $\boldsymbol{s}_{\text {all }}$ are arranged as

$$
\begin{aligned}
& \mathbf{S}_{\text {all }}
\end{aligned}
$$

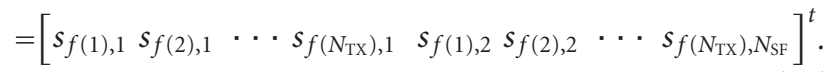

$$
\begin{aligned}
& \mathbf{F}=\left[\begin{array}{cccc}
w_{1,1} \mathbf{H}_{1} & w_{1,2} \mathbf{H}_{1} & \cdots & w_{1, N_{\mathrm{SF}}} \mathbf{H}_{1} \\
w_{2,1} \mathbf{H}_{2} & w_{2,2} \mathbf{H}_{2} & & \vdots \\
\vdots & & \ddots & \\
w_{N_{\mathrm{SF}}, 1} \mathbf{H}_{N_{\mathrm{SF}}} & \cdots & & w_{N_{\mathrm{SF}}, N_{\mathrm{SF}}} \mathbf{H}_{N_{\mathrm{SF}}}
\end{array}\right] \\
& \mathbf{s}_{\mathrm{all}}=\left[\begin{array}{llllllll}
s_{1,1} & s_{2,1} & \cdots & s_{N_{\mathrm{TX}}, 1} & s_{1,2} & s_{2,2} & \cdots & s_{N_{\mathrm{TX}}, N_{\mathrm{SF}}}
\end{array}\right]^{t} .
\end{aligned}
$$

Assuming flat fading such as $\mathbf{H}_{1}=\mathbf{H}_{2}=\cdots=\mathbf{H}_{N_{\mathrm{SF}}}$ for simplicity, $(b-1) N_{\mathrm{TX}}+1$ to $b N_{\mathrm{TX}}$ th column vectors of matrix $\mathbf{F}$ in the form of (21) are correlated to each other, and all the other combinations of column vectors are orthogonal since
The additional use of the received signal power-based ordering in the code-first ordering method can further improve the PER performance of the proposed MLD. However, the gain by using the additional received signal power-based ordering is expected to be small since the symbols transmitted from the same antenna are dispersed over $\mathbf{s}_{\text {all }}$ anyway in the code-first ordering method to give higher priority to reducing the fading correlation between neighbor-ordered symbols.

\section{Simulation Results}

3.1. Simulation Parameters. The PER of the proposed MLD is measured by computer simulation and compared to that 


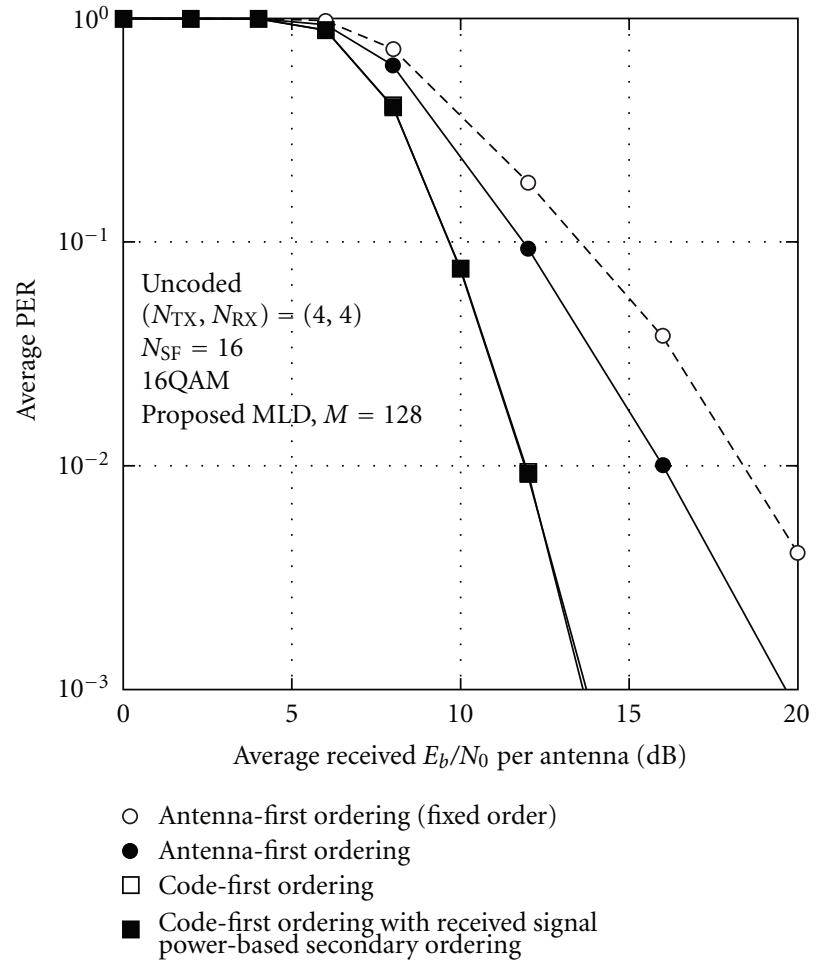

(a) Uncoded case

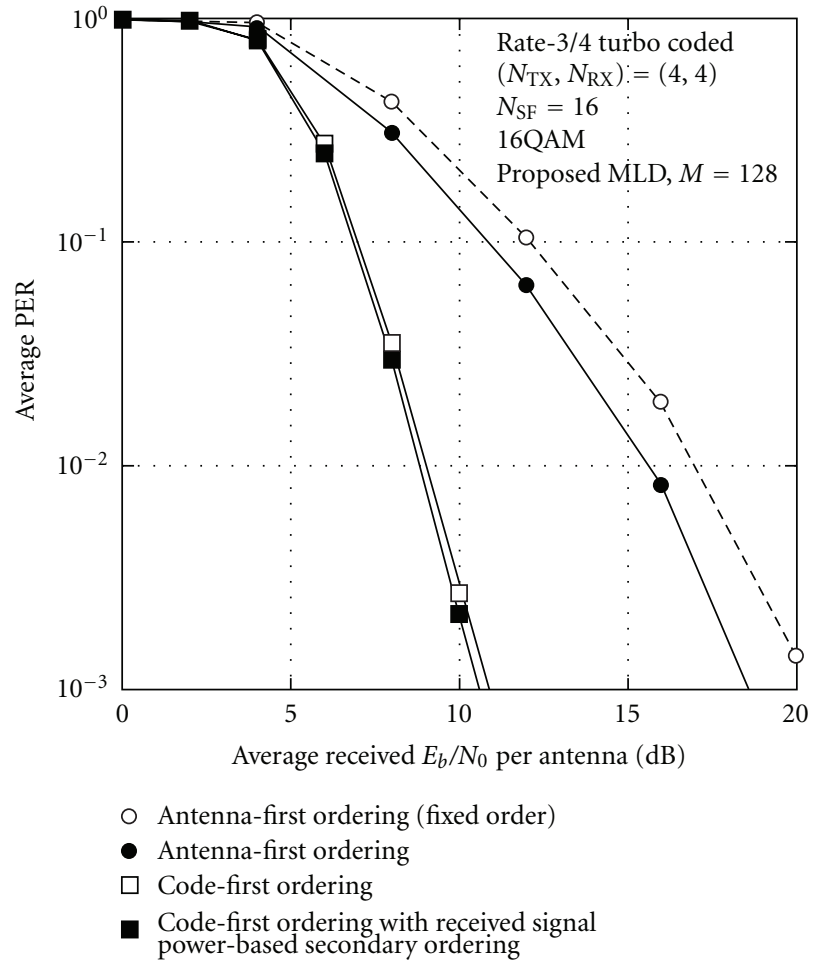

(b) Coded case

FIGURE 4: Comparison of symbol ordering methods.

for the conventional MMSE receiver. Table 1 summarizes the simulation parameters. We assume DFT-spread OFDM, thus the DFT sequence is used as the spreading code. The number of subcarriers that equals the spreading factor, $N_{\mathrm{SF}}$, is parameterized from 4 to 128 . The subcarrier spacing is set to $15 \mathrm{kHz}$. One packet comprises 14 OFDM symbols. As the MIMO configuration, $\left(N_{\mathrm{TX}}, N_{\mathrm{RX}}\right)$ of $(2,2)$ and $(4,4)$ are tested. QPSK and 16QAM are assumed as the data modulation scheme, and the rate-1/2, 3/4, and 8/9 Turbo codes generated by puncturing the rate- $1 / 3$ Turbo code with the constraint length of 4 are used as the channel code. The packet error is assumed to be perfectly detected.

As a channel model, an exponentially decayed 6-path block Rayleigh fading with the rms delay spread of $1 \mu \mathrm{s}$ is assumed where the fading correlation among the transmitter antennas and receiver antennas is zero.

The channel estimation and noise power estimation at the receiver are assumed to be perfect. The LLR calculation method from the branch metrics of the surviving symbol candidates at the last stage of the $M$-algorithm is based on [13]. The Max-Log MAP (maximum a posteriori) decoding with 8 iterations is used for the decoding of the Turbo code.

3.2. Simulation Results. Figures 4(a) and 4(b) show the average PER of the proposed MLD with the antenna-first ordering and code-first ordering methods as a function of the average received signal energy per bit-to-noise spectrum density ratio $\left(E_{b} / N_{0}\right)$ for uncoded and coded cases, respectively. The MIMO configuration $\left(N_{\mathrm{TX}}, N_{\mathrm{RX}}\right)$ is $(4,4)$ and $N_{\mathrm{SF}}$
TABLE 1: Simulation parameters.

\begin{tabular}{|c|c|}
\hline Parameter & Value \\
\hline Modulation & DFT-spread OFDM \\
\hline $\begin{array}{l}N_{\mathrm{SF}}(=\text { number of } \\
\text { subcarriers })\end{array}$ & $4,8,16,32,64$, and 128 \\
\hline Subcarrier spacing & $15 \mathrm{kHz}$ \\
\hline$\left(N_{\mathrm{TX}}, N_{\mathrm{RX}}\right)$ & $(2,2)$ and $(4,4)$ \\
\hline Data modulation & QPSK, 16QAM \\
\hline Channel coding & $\begin{array}{l}\text { Turbo code }(R=1 / 2,3 / 4 \text {, and } 8 / 9) / \text { Max- } \\
\text { Log MAP decoding }\end{array}$ \\
\hline Packet length & 14 OFDM symbols \\
\hline Channel model & $\begin{array}{l}\text { Exponentially decayed 6-path Rayleigh } \\
\text { fading } \\
\text { (rms delay spread }=1 \mu \mathrm{s} \text {, No fading } \\
\text { correlation between antennas) }\end{array}$ \\
\hline Channel estimation & Ideal \\
\hline
\end{tabular}

is 16. 16QAM is used and the rate-3/4 Turbo code is assumed for the coded case. The number $M$ of the surviving symbol candidates for each stage of the $M$-algorithm is set to 128 . As a reference, the antenna-first ordering with fixed antenna order (thus received signal power-independent) is also tested. The PER with code-first ordering with additional use of the received signal power-based ordering is also shown. The effect of adaptive ordering based on the received signal power is observed in the antenna-first ordering method. However, 


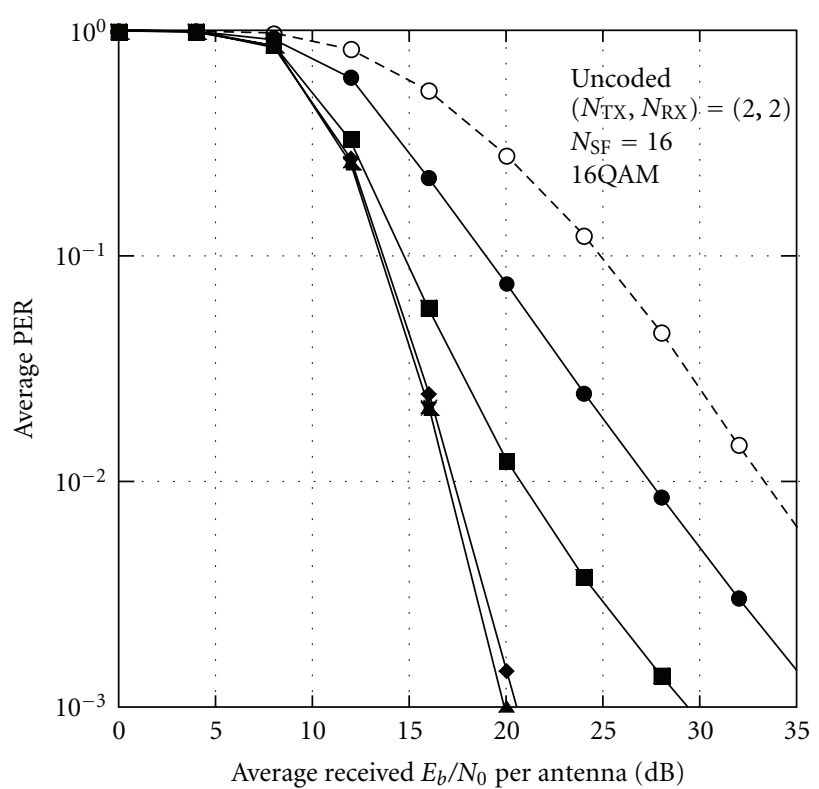

- MMSE

Proposed MLD

(code-first ordering)

- $M=1$

- $M=4$

- $M=16$

\ $M=64$

จ $M=128$

- $M=512$

$\times M=4096$

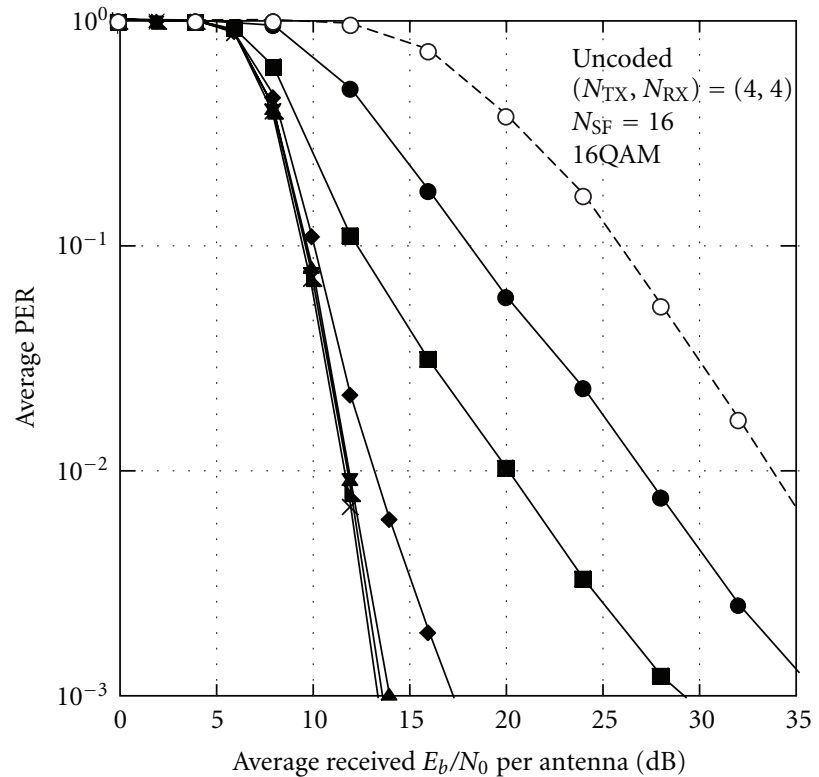

- MMSE

Proposed MLD

(code-first ordering)

- $M=1$

- $M=4$

- $M=16$

\ $M=64$

> $M=128$

- $M=512$

$\times M=4096$

(a) $\left(N_{\mathrm{TX}}, N_{\mathrm{RX}}\right)=(2,2)$

(b) $\left(N_{\mathrm{TX}}, N_{\mathrm{RX}}\right)=(4,4)$

FIgURE 5: Average PER as a function of average received $E_{b} / N_{0}$ (uncoded case).

Figures 4(a) and 4(b) show that the code-first ordering method greatly improves the achievable PER compared to the antenna-first ordering method. This result indicates that in OFDM MIMO multiplexing with frequency domain spreading and code multiplexing, decreasing the fading correlation between neighbor-ordered transmitted symbols is more important than increasing the received signal power for improving the accuracy of the selection of the surviving symbol candidates in the $M$-algorithm. Meanwhile in codefirst ordering, the additional secondary ordering based on the received signal power does not significantly improve the PER. This is because the symbols transmitted from the same antenna are dispersed over the transmit symbol vector anyway in the code-first ordering method to give higher priority to the reduction in the fading correlation between neighbor-ordered symbols. When we compare Figures 4(a) and 4(b), the PER improvement by using the code-first ordering in the coded case is larger than that in the uncoded case. This may indicate that the code-first ordering is effective not only for detecting the ML symbol vector that has least accumulated branch metric but also for finding the other symbol vectors that have relatively low accumulated branch metrics, which is important for calculating an accurate LLR for the coded bits. In the following evaluation, the code-first ordering method is used for the proposed MLD.

Figures 5(a) and 5(b) show the average PER for the uncoded case as a function of the average received $E_{b} / N_{0}$ for $\left(N_{\mathrm{TX}}, N_{\mathrm{RX}}\right)$ of $(2,2)$ and $(4,4)$, respectively. 16QAM is assumed. The number of subcarriers, which is equal to $N_{\mathrm{SF}}$, is set to 16 . In the proposed MLD, the number $M$ of the surviving symbol candidates for each stage of the $M$-algorithm is parameterized from 1 to 4096. For comparison, the PER of the conventional MMSE receiver is also plotted. In Figure 5(a), the required average received $E_{b} / N_{0}$ for the average PER of $10^{-2}$ is significantly reduced according to the increase in the $M$ value. This is because the number of false discards of the correct symbol candidates can be decreased by increasing the $M$ value. We find, nevertheless, that the reduction in the required average $E_{b} / N_{0}$ is small by increasing the $M$ value beyond 16 . When $M$ is 16 , the required average received $E_{b} / N_{0}$ for the average PER of $10^{-2}$ is reduced by approximately $15 \mathrm{~dB}$ compared to the case with conventional MMSE-based filtering. Regarding the computational complexity, while the PER with full MLD and the proposed MLD with $M$ of 64 are expected to be approximately identical, the number of branch metric calculations is reduced from $2^{N_{\mathrm{R}} N_{\mathrm{TX}} N_{\mathrm{SF}}} \approx 3.4 \times 10^{38}$, which is 


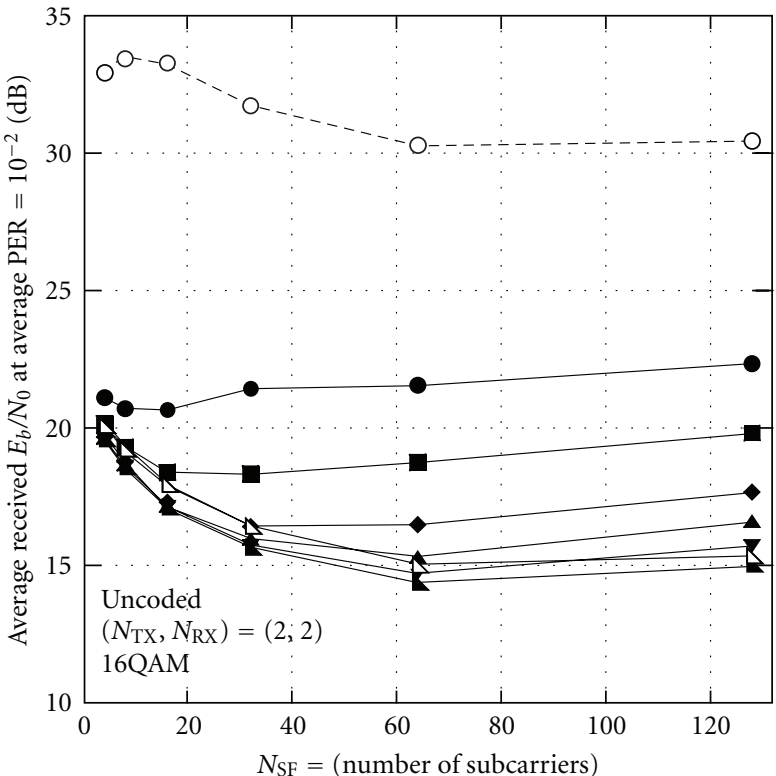

Proposed MLD
(code-first ordering)
- $M=4$
- $M=8$
- $M=16$
A $M=32$
- $M=64$
- $M=128$

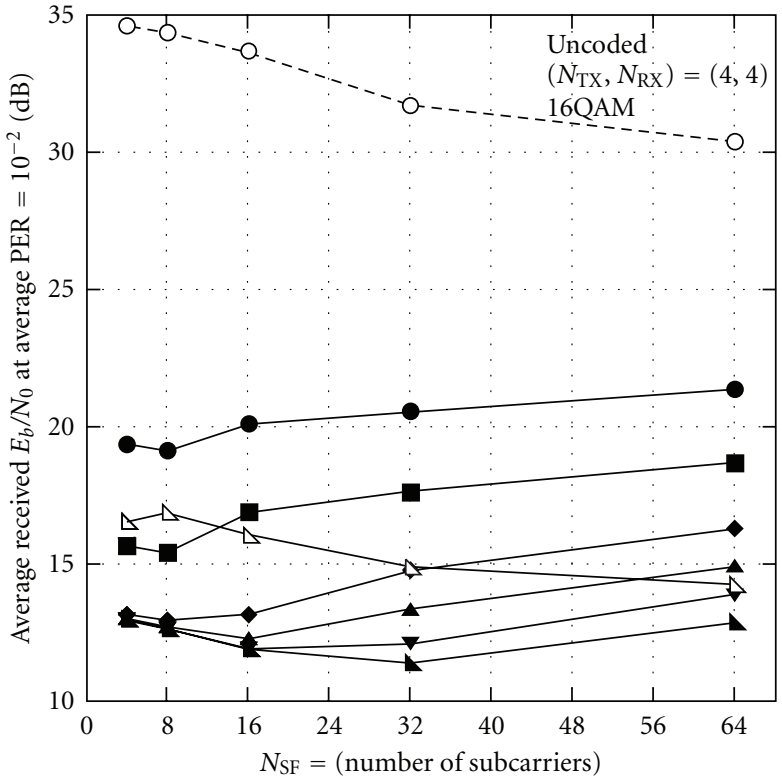

Proposed MLD
(code-first ordering)
- $M=4$
- $M=8$
- $M=16$
- $M=32$
- $M=64$
- $M=128$

O MMSE

Proposed MLD

(antenna-first ordering)

$\triangle M=128$

(a) $\left(N_{\mathrm{TX}}, N_{\mathrm{RX}}\right)=(2,2)$

(b) $\left(N_{\mathrm{TX}}, N_{\mathrm{RX}}\right)=(4,4)$

FiguRE 6: Required average received $E_{b} / N_{0}$ as a function of $N_{\text {SF }}$ (uncoded case).

required for full MLD, to $M 2^{N_{\mathrm{R}}} N_{\mathrm{TX}} N_{\mathrm{SF}} \approx 3.3 \times 10^{4}$ by using the proposed MLD.

In Figure 5(b), approximately the same behavior in the PER performance is observed for $\left(N_{\mathrm{TX}}, N_{\mathrm{RX}}\right)$ of $(4,4)$ as for $(2,2)$. However, as the number of spatially multiplexed symbols is increased, the required $M$ value for achieving a near saturated PER is increased (to approximately 64). Since the proposed MLD achieves receiver antenna diversity that is different from that when using the conventional MMSE receiver, the reduction in the required average received $E_{b} / N_{0}$ for the average PER of $10^{-2}$ by using the proposed MLD with $M$ of 64 compared to the conventional MMSE-based filtering is increased to approximately $22 \mathrm{~dB}$ for $\left(N_{\mathrm{TX}}, N_{\mathrm{RX}}\right)$ of $(4,4)$.

Figures 6(a) and 6(b) show the required average received $E_{b} / N_{0}$ for the average PER of $10^{-2}$ as a function of $N_{\mathrm{SF}}$ for $\left(N_{\mathrm{TX}}, N_{\mathrm{RX}}\right)$ of $(2,2)$ and $(4,4)$, respectively. 16QAM and no channel coding are assumed. In the proposed MLD, $M$ is parameterized from 4 to 128 . For comparison, the required average received $E_{b} / N_{0}$ of the conventional MMSE receiver and that of the proposed MLD with antenna-first ordering and $M$ of 128 are also plotted. The reason why the required average received $E_{b} / N_{0}$ of the conventional MMSE receiver is decreased according to the increase in $N_{\mathrm{SF}}$ (= number of subcarriers) is the increased frequency diversity. Meanwhile, the performance improvement due to the increased frequency diversity is small in the proposed MLD especially for $\left(N_{\mathrm{TX}}, N_{\mathrm{RX}}\right)$ of $(4,4)$. This is because the proposed MLD achieves receiver diversity; therefore, the additional diversity gain via frequency diversity is small. Furthermore, as $N_{\mathrm{SF}}$ increases, the number of false discards of the correct symbol candidates is increased in the $M$-algorithm of the proposed MLD especially at the earlier stages since the signal energy per stage is reduced as the number of stages in the $M$-algorithm is proportional to the $N_{\mathrm{SF}}$ value. However, even in a relatively large $N_{\mathrm{SF}}$ case such as 64 , the proposed MLD with the $M$ of 128 can reduce the required average received $E_{b} / N_{0}$ for the average PER of $10^{-2}$ by approximately $17.5 \mathrm{~dB}$ compared to the conventional MMSE receiver. We can also see that the performance enhancement by using the codefirst ordering method compared to the antenna-first one is more significant as $N_{\mathrm{SF}}$ decreases. This is because when $N_{\mathrm{SF}}$ is small, average fading correlation between $\mathbf{H}_{i}$ becomes larger.

Figures 7(a) and 7(b) show the average PER assuming rate-3/4 Turbo coding as a function of the average received $E_{b} / N_{0}$ for $\left(N_{\mathrm{TX}}, N_{\mathrm{RX}}\right)$ of $(2,2)$ and $(4,4)$, respectively, with $M$ as a parameter. $N_{\mathrm{SF}}$ is set to $16.16 \mathrm{QAM}$ is assumed. For comparison, the PER of the conventional MMSE receiver is also plotted. Compared to the uncoded case shown in Figures 5(a) and 5(b), the PER performance both for the proposed MLD and conventional MMSE receivers is improved. Since 

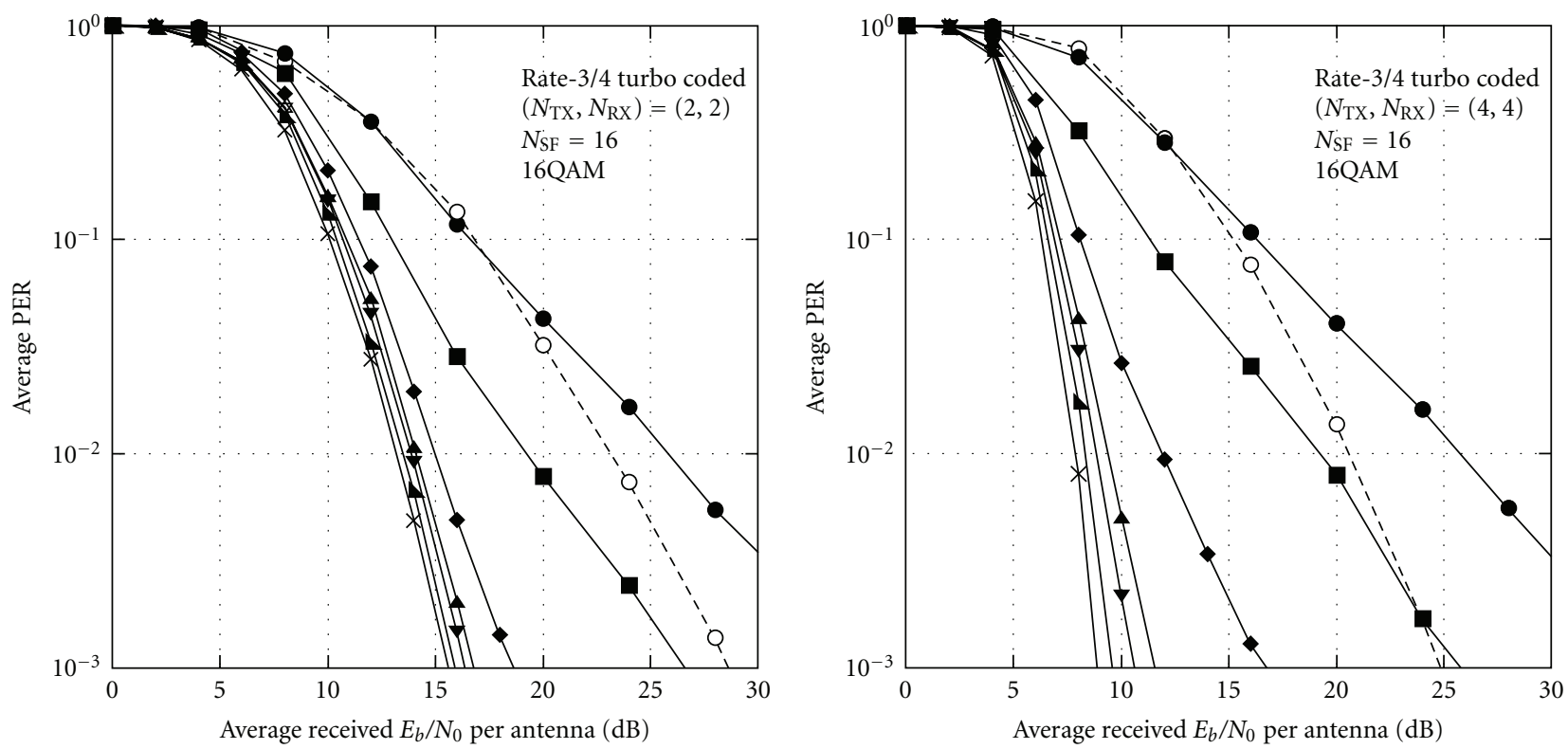

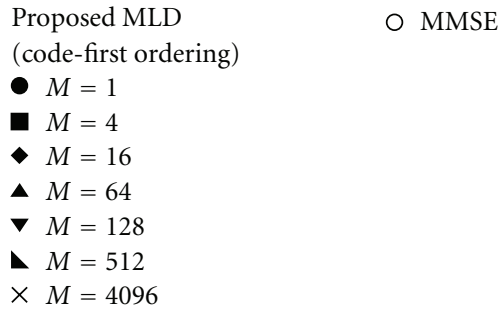

(a) $\left(N_{\mathrm{TX}}, N_{\mathrm{RX}}\right)=(2,2)$

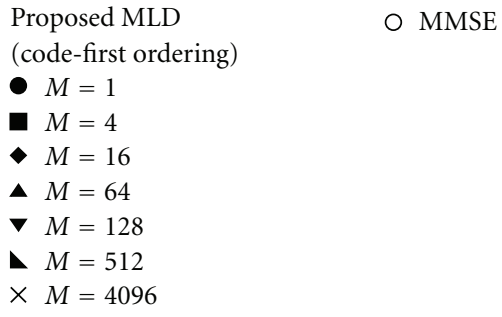

(b) $\left(N_{\mathrm{TX}}, N_{\mathrm{RX}}\right)=(4,4)$

FIgURE 7: Average PER as a function of average received $E_{b} / N_{0}$ (coded case).

the conventional MMSE receivers can achieve some degree of diversity gain during the channel decoding, the performance improvement of the conventional MMSE receivers is larger than that of the proposed MLD receiver. As a result, the PER reduction effect by using the proposed MLD compared to the conventional MMSE receiver is decreased when channel coding is applied. However, the required average received $E_{b} / N_{0}$ for the average PER of $10^{-2}$ is still significantly reduced when the proposed MLD is assumed due to the large receiver antenna diversity gain even with the channel coding. When $M$ is 128 , the required average received $E_{b} / N_{0}$ for the average PER of $10^{-2}$ is reduced by approximately $9 \mathrm{~dB}$ compared to the case with conventional MMSE-based filtering for $\left(N_{\mathrm{TX}}, N_{\mathrm{RX}}\right)$ of $(2,2)$. Since the proposed MLD achieves receiver antenna diversity that is different from that when using the conventional MMSE receiver, the reduction in the required average received $E_{b} / N_{0}$ for the average PER of $10^{-2}$ by using the proposed MLD with $M$ of 128 compared to the conventional MMSE-based filtering is increased to approximately $12 \mathrm{~dB}$ for $\left(N_{\mathrm{TX}}, N_{\mathrm{RX}}\right)$ of $(4,4)$. The required $M$ value for achieving a near saturated PER in OFDM MIMO multiplexing with frequency domain spreading and code multiplexing is larger than that for OFDM MIMO multiplexing without spreading, for example, in [12, 13]. This is because the use of the code multiplexing increases the number of symbol candidates to be tested. Furthermore, the use of the code multiplexing also increases the number of stages in the $M$-algorithm from $N_{\mathrm{TX}}$ to $N_{\mathrm{TX}} N_{\mathrm{SF}}$, which results in reduced signal energy per stage.

Figures $8(\mathrm{a})$ and 8 (b) show the required average received $E_{b} / N_{0}$ for the average PER of $10^{-2}$ assuming rate-3/4 Turbo coding as a function of $N_{\mathrm{SF}}$ for $\left(N_{\mathrm{TX}}, N_{\mathrm{RX}}\right)$ of $(2,2)$ and $(4$, 4 ), respectively. 16QAM is assumed. In the proposed MLD, $M$ is parameterized from 16 to 512 . For comparison, the required average received $E_{b} / N_{0}$ of the conventional MMSE receiver and that of the proposed MLD with antenna-first ordering and the $M$ of 128 are also plotted. Basically the same performance tendency is observed as in Figures 6(a) and $6(\mathrm{~b})$. Although the number of false discards of the correct symbol candidates is increased in the $M$-algorithm of the proposed MLD as $N_{\mathrm{SF}}$ increases, even in a relatively large $N_{\mathrm{SF}}$ case such as 64 , the proposed MLD with the $M$ of $128 \mathrm{can}$ reduce the required average received $E_{b} / N_{0}$ for the average PER of $10^{-2}$ by approximately $5 \mathrm{~dB}$ compared to the conventional MMSE receiver for $\left(N_{\mathrm{TX}}, N_{\mathrm{RX}}\right)$ of $(4,4)$.

Figures 9(a)-9(d) show the average PER assuming various modulation and channel coding rates as a function of the average received $E_{b} / N_{0}$, with $M$ as a parameter. Figures 9(a) and 9(b) assume QPSK data modulation with the Turbo code rate of $1 / 2$ and $8 / 9$, respectively. Figures $9(\mathrm{c})$ and 


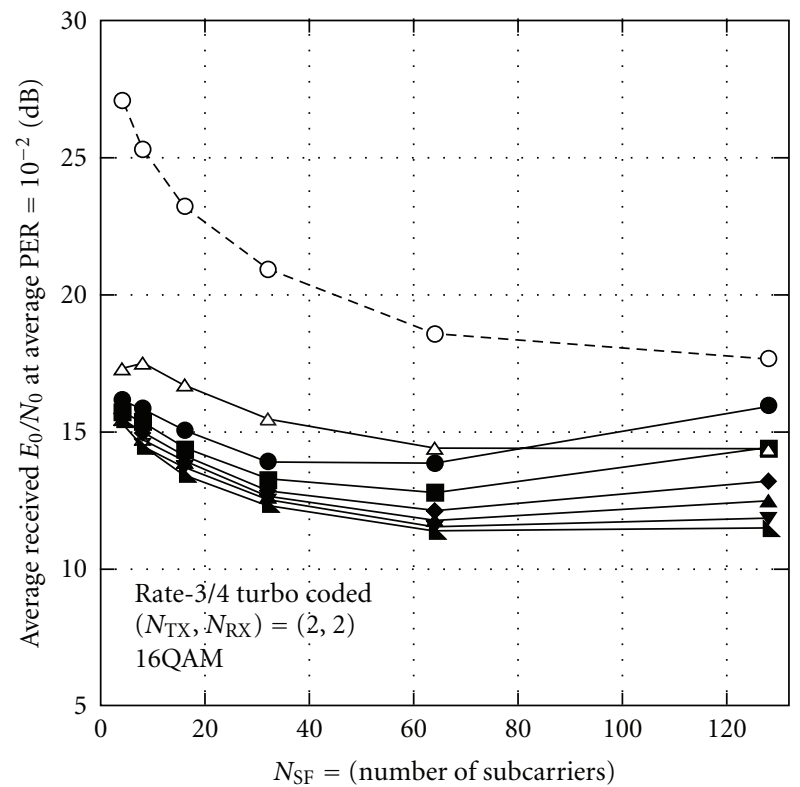

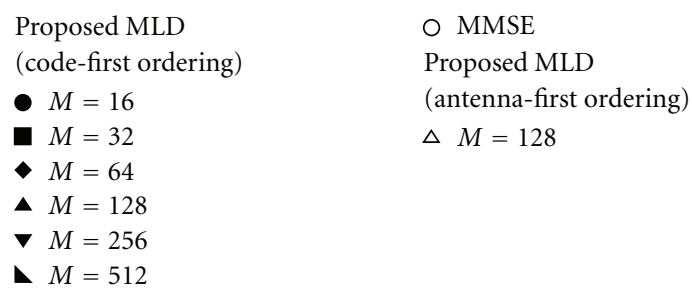

(a) $\left(N_{\mathrm{TX}}, N_{\mathrm{RX}}\right)=(2,2)$

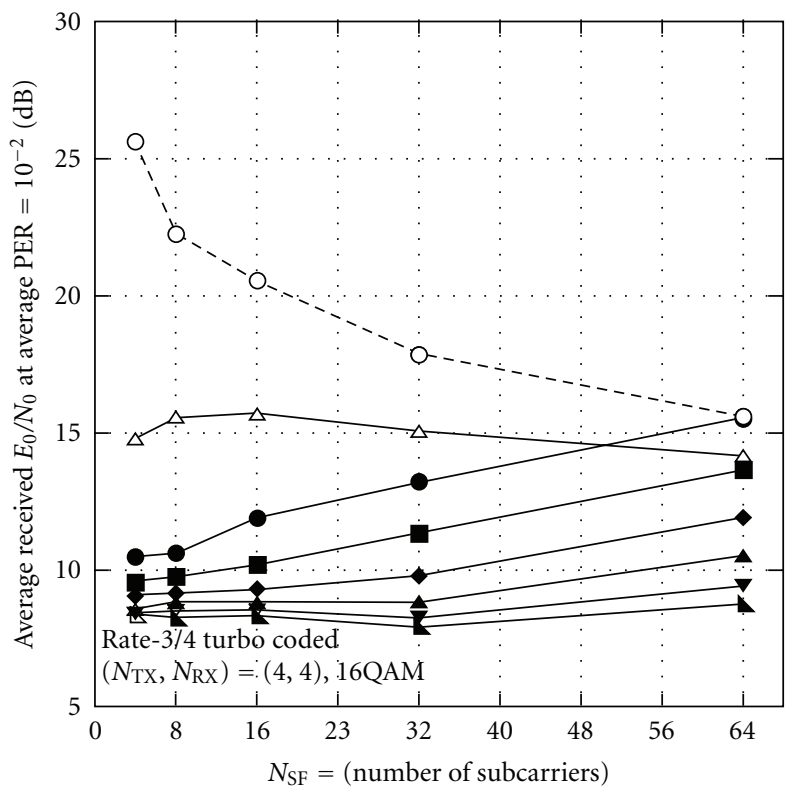

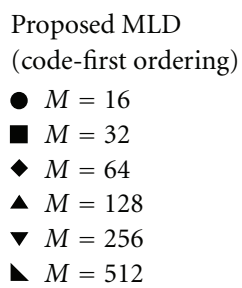

O MMSE

Proposed MLD (antenna-first ordering) $\Delta M=128$ (b) $\left(N_{\mathrm{TX}}, N_{\mathrm{RX}}\right)=(4,4)$

FIGURE 8: Required average received $E_{b} / N_{0}$ as a function of $N_{\mathrm{SF}}$ (coded case).

9(d) assume 16QAM data modulation with the Turbo code rate of $1 / 2$ and $8 / 9$, respectively. The MIMO configuration $\left(N_{\mathrm{TX}}, N_{\mathrm{RX}}\right)$ is $(4,4)$ and $N_{\mathrm{SF}}$ is 16 . For comparison, the PER of the conventional MMSE receiver is also plotted. From Figure 9(a), we see that the gain in the required $E_{b} / N_{0}$ for the average PER of $10^{-2}$ by using the proposed MLD compared to the conventional MMSE receiver is not so significant when QPSK modulation with the Turbo code rate of $1 / 2$ is assumed. This is because the use of QPSK reduces the operating point of the average received $E_{b} / N_{0}$, which reduces the diversity gain by using the MLD-based signal detection, and the use of a lower coding rate along with channel coding across the transmitter antenna mitigates the degraded diversity gain in the MMSE-based filtering during the channel decoding process. This also explains the reason why the PER with the conventional MMSE-based filtering is more dependent on the coding rate than that with the proposed MLD-based detection. However, we also see that the gain of the proposed MLD over the conventional MMSE-based filtering is enhanced according to the use of the higher order modulation and coding rate. This means that the proposed MLD is effective in achieving a very high frequency efficiency by using MIMO multiplexing with a high-order modulation and coding rate for OFDM with frequency domain spreading and code multiplexing, similar to the case with OFDM without spreading $[21,22]$.
We evaluate the computational complexity of the proposed MLD from the viewpoint of the required number of real multiplications per symbol. Table 2 gives the required number of real multiplications per symbol. For comparison, the computational complexity levels of the full MLD and the MMSE-based filtering are also evaluated. For all methods, the computational complexity required for the time/frequency synchronization and channel estimation are not taken into account since they are common to all methods and the complexity of these processes is largely dependent on the applied algorithms. In Table 2, we assume that $N_{\mathrm{TX}}$ is equal to $N_{\mathrm{RX}}$ and they are denoted as $N_{\mathrm{ANT}}=N_{\mathrm{TX}}=$ $N_{\mathrm{RX}}$. Term $C$, which represents the number of constellation points, is equal to $2^{N_{\mathrm{R}}}$; thus $C$ is 4 and 16 , for QPSK and 16QAM, respectively. From the table, the proposed MLD can significantly reduce the computational complexity more than the full MLD, assuming $N_{\mathrm{ANT}}=4, C=16, N_{\mathrm{SF}}=16$, and $M=$ 128. The computational complexity of the proposed MLD is approximately 70 times higher than that for the conventional MMSE-based filtering. From the table, the computational complexity of the proposed MLD is dominated by the QR decomposition of the matrix $\mathbf{F}$ and the calculation of the squared Euclidian distances although the number of squared Euclidian distance calculations is significantly reduced compared to the full MLD. Therefore, for further study, we can consider two approaches to reduce further 

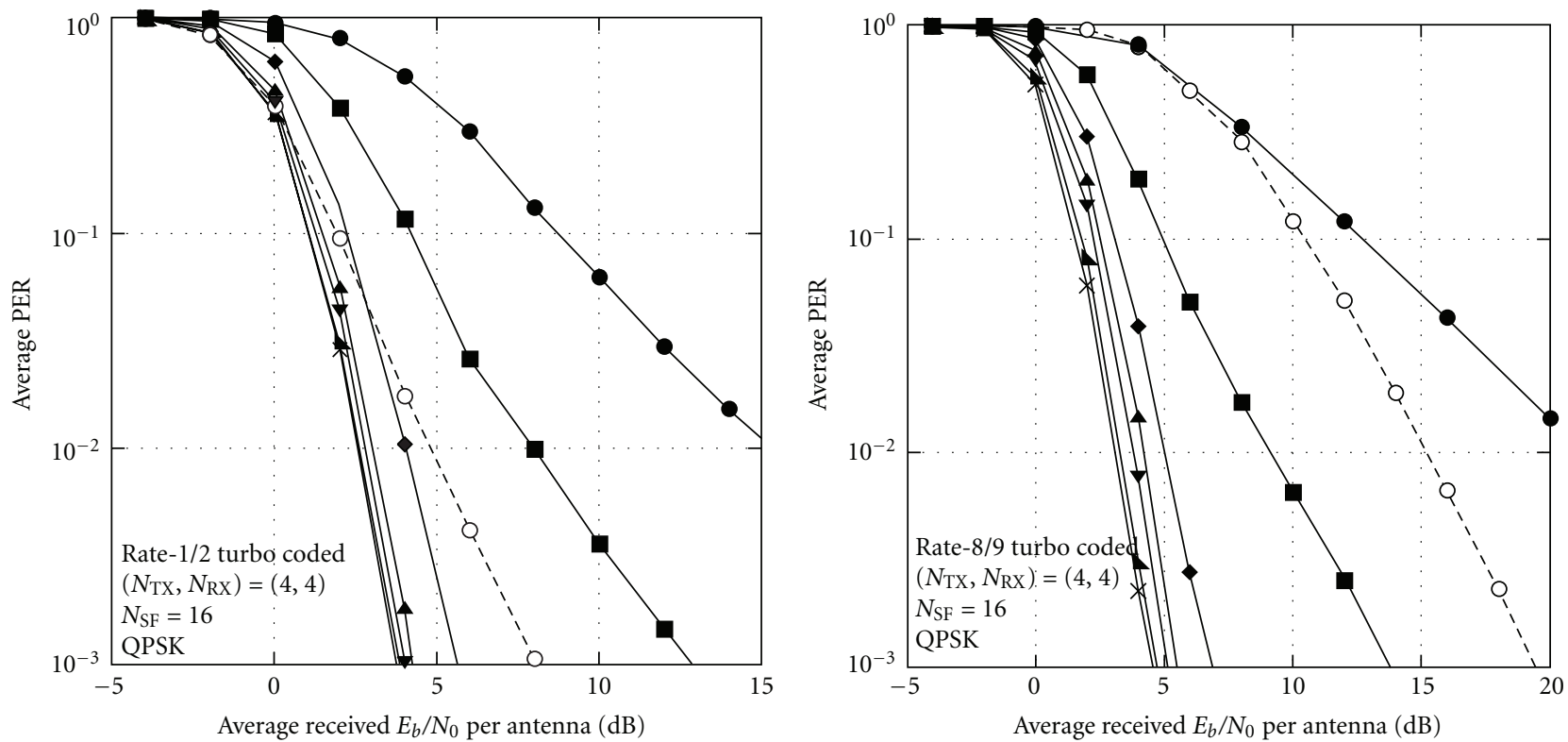

- MMSE

Proposed MLD

(code-first ordering)

- $M=1 \quad \nabla M=128$

- $M=4 \quad$ \ $M=512$

- $M=16 \times M=4096$

\ $M=64$

(a) QPSK, rate-1/2 Turbo coded

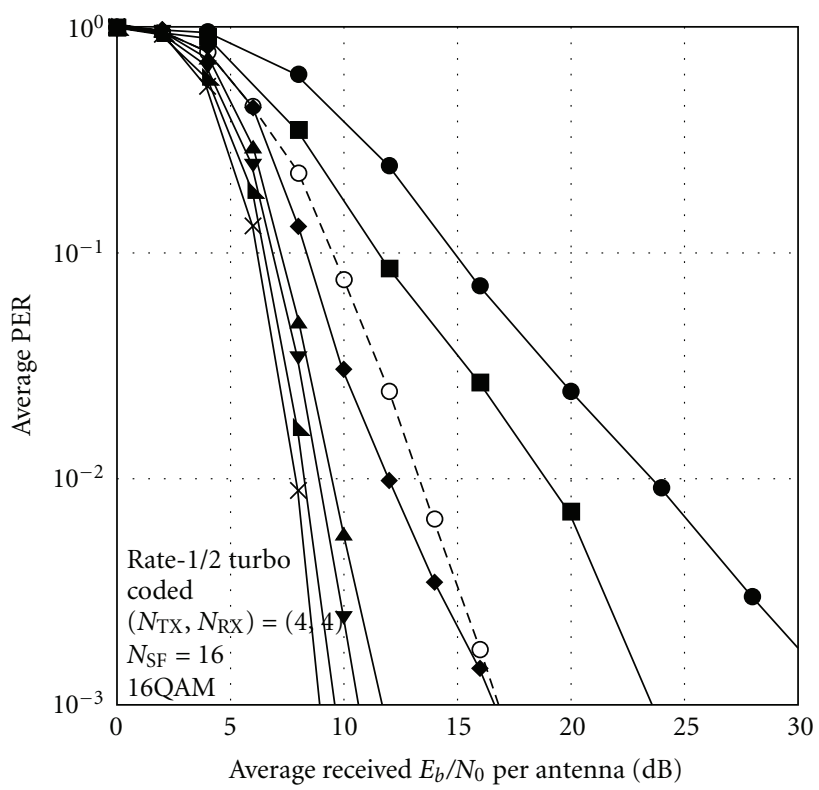

- MMSE

Proposed MLD

(code-first ordering)

- $M=1 \quad \nabla M=128$

- $M=4 \quad \triangle M=512$

- $M=16 \times M=4096$

\ $M=64$

(b) QPSK, rate-8/9 Turbo coded

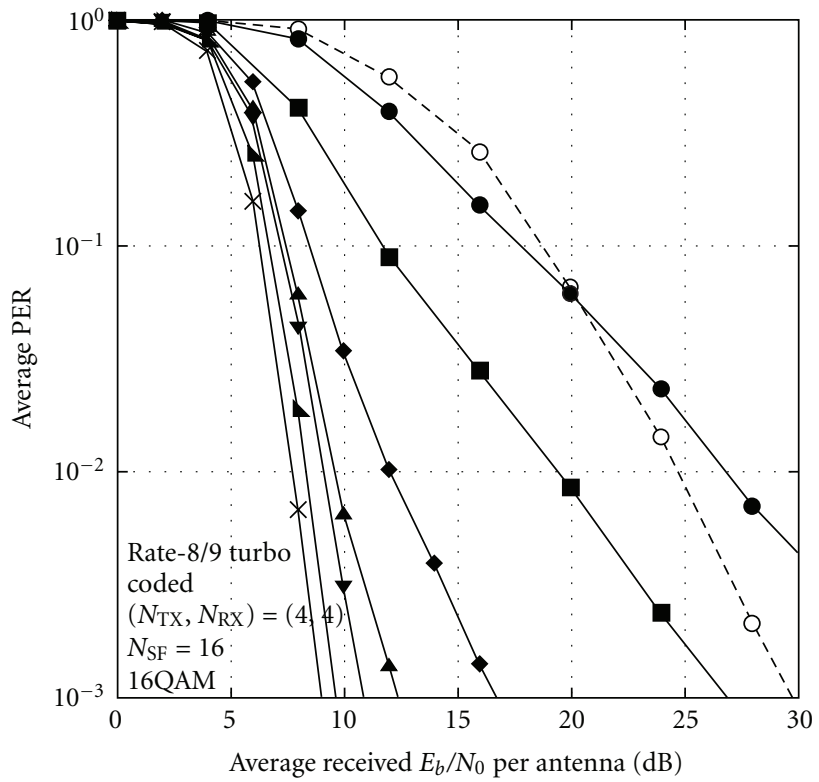

- MMSE

Proposed MLD

(code-first ordering)

- $M=1 \quad \nabla M=128$

- $M=4$ \ $M=512$

- $M=16 \times M=4096$

\ $M=64$

(c) 16QAM, rate-1/2 Turbo coded

- MMSE

Proposed MLD

(code-first ordering)

- $M=1 \quad \nabla M=128$

- $M=4$ \ $M=512$

- $M=16 \times M=4096$

\ $M=64$

(d) 16QAM, rate-8/9 Turbo coded

FIGURE 9: Average PER as a function of average received $E_{b} / N_{0}$ for various modulation schemes and coding rates. 
TABLE 2: Number of real multiplications per symbol required for signal detection.

\begin{tabular}{|c|c|c|c|}
\hline Signal detection method & Process & $\begin{array}{l}\text { Required number of real } \\
\text { multiplications }\end{array}$ & $\begin{array}{l}\text { Example: } N_{\mathrm{ANT}}=4, \\
C=16, N_{\mathrm{SF}}=16, M=128\end{array}$ \\
\hline \multirow{4}{*}{ Full MLD } & FFT & $4 N_{\mathrm{ANT}} N_{\mathrm{SF}} \log _{2} N_{\mathrm{SF}}$ & 1,024 \\
\hline & Generation of symbol replica candidates & $4 N_{\mathrm{ANT}}^{2} C N_{\mathrm{SF}}^{2}$ & 262,144 \\
\hline & Calculation of squared Euclidian distances & $2 N_{\mathrm{ANT}} C^{N_{\mathrm{ANT}} N_{\mathrm{SF}}} N_{\mathrm{SF}}$ & $1.482 \times 10^{79}$ \\
\hline & Total & & $1.482 \times 10^{79}$ \\
\hline \multirow{6}{*}{ MMSE-based filtering } & FFT & $4 N_{\mathrm{ANT}} N_{\mathrm{SF}} \log _{2} N_{\mathrm{SF}}$ & 1,024 \\
\hline & MMSE weight generation & $12 N_{\mathrm{ANT}}^{3} N_{\mathrm{SF}}$ & 12,288 \\
\hline & MMSE weight multiplication & $4 N_{\mathrm{ANT}}^{2} N_{\mathrm{SF}}$ & 1,024 \\
\hline & Despreading & $4 N_{\mathrm{ANT}} N_{\mathrm{SF}}^{2}$ & 4,096 \\
\hline & Calculation of squared Euclidian distances & $2 N_{\mathrm{ANT}} C N_{\mathrm{SF}}$ & 2,048 \\
\hline & Total & & 20,480 \\
\hline \multirow{7}{*}{ Proposed MLD } & FFT & $4 N_{\mathrm{ANT}} N_{\mathrm{SF}} \log _{2} N_{\mathrm{SF}}$ & 1,024 \\
\hline & Generation of matrix $\mathbf{F}$ & $4 N_{\mathrm{ANT}} N_{\mathrm{SF}}^{2}$ & 4,096 \\
\hline & QR decomposition of matrix $\mathbf{F}$ & $4 N_{\mathrm{ANT}}^{3} N_{\mathrm{SF}}^{3}+8 N_{\mathrm{ANT}}^{2} N_{\mathrm{SF}}^{2}$ & $1,081,344$ \\
\hline & Multiplication of $\mathbf{Q}^{H}$ to received signal vector & $4 N_{\mathrm{ANT}}^{2} N_{\mathrm{SF}}^{2}$ & 16,384 \\
\hline & Generation of symbol replica candidates & $\begin{array}{l}4\left(N_{\mathrm{ANT}} N_{\mathrm{SF}}\left(N_{\mathrm{ANT}} N_{\mathrm{SF}}\right.\right. \\
+1) / 2) C\end{array}$ & 133,120 \\
\hline & Calculation of squared Euclidian distances & $2 N_{\mathrm{ANT}} C N_{\mathrm{SF}} M$ & 262,144 \\
\hline & Total & & $1,498,112$ \\
\hline
\end{tabular}

the computational complexity of the proposed MLD. The first one is complexity reduction in the QR decomposition of the matrix F. By utilizing the special structure of matrix $\mathbf{F}$, there is a possibility to reduce the calculation cost of the $\mathrm{QR}$ decomposition (we assume that the inner product calculation in the Gram-Schmidt orthogonalization can be simplified). The second approach is to reduce the complexity in the calculation of the squared Euclidian distances. For example, by applying the method described in [22-24], the computational complexity of the process for squared Euclidian distance calculations will be reduced without PER performance degradation.

Figure 10 shows the required number of real multiplications for different modulation schemes with the Turbo code rate of $3 / 4$ as a function of the required average received $E_{b} / N_{0}$ for the average PER of $10^{-2}$. The MIMO configuration $\left(N_{\mathrm{TX}}, N_{\mathrm{RX}}\right)$ is $(4,4)$ and $N_{\mathrm{SF}}$ is 16 . The relationship between the required number of real multiplications and required average received $E_{b} / N_{0}$ in the proposed MLD is varied by changing the $M$ value. We see that the proposed MLD can reduce the required average received $E_{b} / N_{0}$ for the average PER of $10^{-2}$ for 16QAM with the rate-3/4 Turbo code by approximately $12 \mathrm{~dB}$ compared to the conventional MMSE receiver at the cost of a 70 times higher computational complexity.

\section{Conclusion}

This paper presented a new MLD-based signal detection method for OFDM MIMO multiplexing with frequency domain spreading and code multiplexing. The proposed MLD-based signal detection method is based on the QRD-M algorithm (or QRM-MLD) for OFDM MIMO multiplexing, which uses per subcarrier-based signal orthogonalization and the computationally efficient $M$-algorithm to decompose the spatially multiplexed transmit symbols. However, the proposed MLD receiver jointly considers all the subcarriers to which the spread symbols are mapped and constructs the overall frequency-domain linear transformation matrix which is a product of the space and frequency-domain channel matrix and spreading code matrix in order to decompose fully the spatial and code multiplexed transmit symbols at the receiver. The $\mathrm{QR}$ decomposition of the overall frequency-domain linear transformation matrix is performed to derive the orthogonalized received signal vector. Then, the $M$-algorithm is used to achieve computationally efficient quasi-MLD with the orthogonalized received signal vector. Furthermore, we showed that when frequency domain spreading and code multiplexing are used in OFDM, the symbol ordering for sequential signal detection based on the fading correlation among the transmitted symbols, which we call code-first ordering, significantly improves the achievable PER performance. Simulation results showed that when the spreading factor and number of code multiplexed symbols are 16, the proposed MLD reduces the required average received $E_{b} / N_{0}$ for the average PER of $10^{-2}$ by approximately 9 and $12 \mathrm{~dB}$ compared to the conventional MMSE-based filtering for 2-by-2 and 4-by-4 MIMO multiplexing, respectively (16QAM with the rate-3/4 Turbo code is assumed).

\section{Acknowledgment}

The authors would like to thank the reviewers for their insightful and constructive suggestions. 


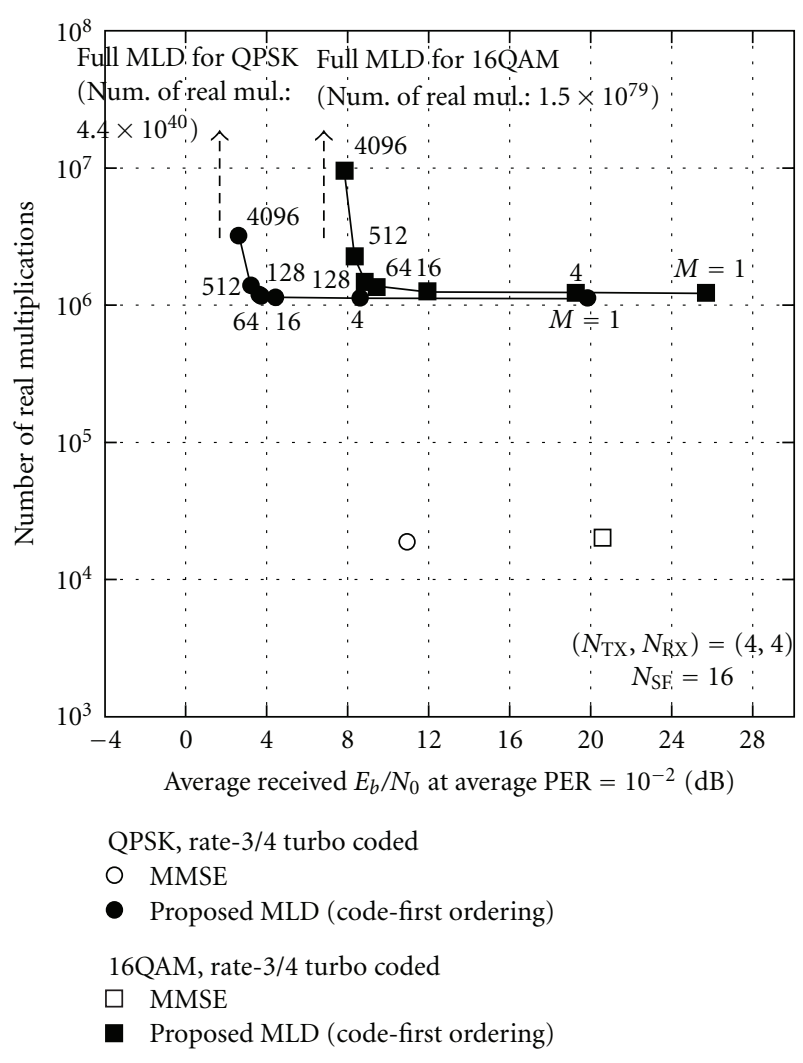

FIGURE 10: Number of real multiplications as a function of required average received $E_{b} / N_{0}$.

\section{References}

[1] 3GPP TS36.300, "Evolved Universal Terrestrial Radio Access (E-UTRA) and Evolved Universal Terrestrial Radio Access Network (E-UTRAN)," Overall description.

[2] N. Yee, J.-P. Linnartz, and G. Fettweis, "Multi-carrier CDMA in indoor wireless radio networks," in Proceedings of the 4th IEEE International Symposium on Personal, Indoor and Mobile Radio Communications (PIMRC'93), pp. 109-113, Yokohama, Japan, September 1993.

[3] K. Fazel and L. Papke, "On the performance of convolutionalcoded CDMA/OFDM for mobile communication systems," in Proceedings of the 4th IEEE International Symposium on Personal, Indoor and Mobile Radio Communications (PIMRC '93), pp. 468-472, Yokohama, Japan, September 1993.

[4] S. Hara and R. Prasad, "Overview of multicarrier CDMA," IEEE Communications Magazine, vol. 35, no. 12, pp. 126-133, 1997.

[5] H. Atarashi, S. Abeta, and M. Sawahashi, "Broadband packet wireless access appropriate for high-speed and high-capacity throughput," in Proceedings of the 53rd Vehicular Technology Conference (VTC '01), vol. 1, pp. 566-570, Rhodos, Greece, May 2001.

[6] Y. Wu, C. K. Ho, and S. Sun, "On some properties of WalshHadamard transformed OFDM," in Proceedings of the 56th Vehicular Technology Conference (VTC '02), pp. 2096-2100, Vancouver, Canada, September 2002.

[7] Y. Wang, X. Tao, P. Zhang, J. Xu, X. Wang, and T. Suzuki, "MIMO-OFDM PAPR reduction by combining shifting and inversion with matrix transform," in Proceedings of the 18th
Annual IEEE International Symposium on Personal, Indoor and Mobile Radio Communications (PIMRC '07), Athens, Greece, September 2007.

[8] D. Galda, H. Rohling, E. Costa, H. Haas, and E. Schulz, "A low complexity transmitter structure for OFDM-FDMA uplink systems," in Proceedings of the 55th Vehicular Technology Conference (VTC'02), pp. 1737-1741, Birmingham, Ala, USA, May 2002.

[9] R. Dinis, D. Falconer, C. T. Lam, and M. Sabbaghian, "A multiple access scheme for the uplink of broadband wireless systems," in Proceedings of IEEE Global Telecommunications Conference (GLOBECOM '04), pp. 3808-3812, Dallas, Tex, USA, December 2004.

[10] G. J. Foschini and M. J. Gans, "On limits of wireless communications in a fading environment when using multiple Antennas," Wireless Personal Communications, vol. 6, no. 3, pp. 311-335, 1998.

[11] A. van Zelst, R. van Nee, and G. A. Awater, "Space division multiplexing (SDM) for OFDM systems," in Proceedings of the 51st IEEE Vehicular Technology Conference (VTC '00), vol. 2, pp. 1070-1074, Tokyo, Japan, May 2000.

[12] K. J. Kim, J. Yue, R. A. Iltis, and J. D. Gibson, "A QRD$\mathrm{M} /$ Kalman filter-based detection and channel estimation algorithm for MIMO-OFDM systems," IEEE Transactions on Wireless Communications, vol. 4, no. 2, pp. 710-721, 2005.

[13] H. Kawai, K. Higuchi, N. Maeda et al., "Likelihood function for QRM-MLD suitable for soft-decision turbo decoding and its performance for OFCDM MIMO multiplexing in multipath fading channel," IEICE Transactions on Communications, vol. E88-B, no. 1, pp. 47-57, 2005.

[14] D. Reynolds and X. Wang, "Low-complexity turbo-equalization for diversity channels," Signal Processing, vol. 81, no. 5, pp. 989-995, 2001.

[15] M. Tüchler, R. Koetter, and A. C. Singer, "Turbo equalization: principles and new results," IEEE Transactions on Communications, vol. 50, no. 5, pp. 754-767, 2002.

[16] T. Abe and T. Matsumoto, "Space-time turbo equalization in frequency-selective MIMO channels," IEEE Transactions on Vehicular Technology, vol. 52, no. 3, pp. 469-475, 2003.

[17] M. Sabbaghian and D. D. Falconer, "An analytical approach for finite block length performance analysis of turbo frequencydomain equalization," IEEE Transactions on Vehicular Technology, vol. 58, no. 3, pp. 1292-1301, 2009.

[18] B. M. Hochwald and S. ten Brink, "Achieving near-capacity on a multiple-antenna channel," IEEE Transactions on Communications, vol. 51, no. 3, pp. 389-399, 2003.

[19] S. Bäro, J. Hagenauer, and M. Witzke, "Iterative detection of mimo transmission using a list-sequential (LISS) detector," in Proceedings of International Conference on Communications (ICC'03), vol. 4, pp. 2653-2657, Anchorage, Alaska, USA, May 2003.

[20] S. Sun, Y. Dai, Z. Lei, H. Kenichi, and H. Kawai, "Pseudoinverse MMSE based QRD-M algorithm for MIMO OFDM," in Proceedings of the 63rd Vehicular Technology Conference (VTC '06), vol. 3, pp. 1545-1549, Melbourne, Australia, MayJuly 2006.

[21] H. Taoka, K. Dai, K. Higuchi, and M. Sawahashi, "Field experiments on MIMO multiplexing with peak frequency efficiency of $50 \mathrm{bit} / \mathrm{second} / \mathrm{Hz}$ using MLD based signal detection for OFDM high-speed packet access," IEEE Journal on Selected Areas in Communications, vol. 26, no. 6, pp. 845-856, 2008.

[22] K. Higuchi, H. Kawai, H. Taoka, N. Maeda, and M. Sawahashi, "Adaptive selection of surviving symbol replica candidates for quasi-maximum likelihood detection using $\mathrm{M}$-algorithm with 
QR-decomposition for OFDM MIMO Multiplexing," IEICE Transactions on Communications, vol. E92-B, no. 4, pp. 12581271, 2009.

[23] H. Kawai, K. Higuchi, N. Maeda, and M. Sawahashi, "Adaptive control of surviving symbol replica candidates in QRM-MLD for OFDM MIMO multiplexing," IEEE Journal on Selected Areas in Communications, vol. 24, no. 6, pp. 1130-1140, 2006.

[24] B.-S. Kim and K. Choi, "A very low complexity QRD-M algorithm based on limited tree search for MIMO systems," in Proceedings of the 67th Vehicular Technology Conference (VTC '08), pp. 1246-1250, Marina Bay, Singapore, May 2008. 